\title{
FEDOSOV QUANTIZATION IN POSITIVE CHARACTERISTIC
}

\author{
R. BEZRUKAVNIKOV AND D. KALEDIN
}

\section{INTRODUCTION}

The so-called deformation quantization of symplectic manifolds originally appeared in $C^{\infty}$ symplectic geometry; the crucial breakthrough was independently made in the early 1980 s by B. Fedosov and M. De Wilde-P. Lecomte. The input of a deformation quantization problem is a symplectic (or, more generally, a Poisson) manifold $M$; the output is a non-commutative one-parameter deformation of the algebra of functions on $M$. Both Fedosov and De Wilde-Lecomte provided general procedures which solve the deformation quantization problem for $C^{\infty}$ manifolds. Recently, motivated in part by M. Kontsevich $[\mathrm{K}]$, there has been much interest in generalizing the deformation quantization procedures to the case of algebraic manifolds equipped with an algebraic symplectic form (see, e.g., [BK1], [Y], and an earlier paper $[\mathrm{NT}]$ for the holomorphic situation). In particular, in [BK1] it has been shown that under some mild assumptions on the manifold, the Fedosov quantization can be made to work in the algebraic setting.

The present paper is a continuation of [BK1] (to which we refer the reader for a more complete bibliography and historical discussion). Namely, one of the most important assumptions in [BK1] (as well as in other papers on the subject) was that the field of definition for all algebraic manifolds has characteristic 0 . In this paper, we study what happens in the case of positive characteristic.

The most obvious new feature of the theory in positive characteristic is the presence of a large Poisson center in the sheaf $\mathcal{O}_{X}$ of functions on a Poisson manifold $X$ : since for any two local functions $f, g \in \mathcal{O}_{X}$ and any Poisson bracket $\{-,-\}$ on $\mathcal{O}_{X}$ we have $\left\{f^{p}, g\right\}=0$, the image $\mathcal{O}_{X}^{p} \subset \mathcal{O}_{X}$ of the Frobenius map lies in the center of any Poisson structure. This phenomenon, already observed in [BMR], allows for interesting applications (see, e.g., [BK2]) but makes the quantization procedures more involved. In this paper, we were not able to prove any meaningful results for general quantizations in positive characteristic, and we had to restrict our attention to a special class of them: the so-called Frobenius-constant quantization. Roughly speaking - the precise definition is Definition 1.4 below - a quantization is Frobenius-constant if the Poisson center $\mathcal{O}_{X}^{p} \subset \mathcal{O}_{X}$ stays central in the quantized algebra $\mathcal{O}_{h}$.

For quantization of this type, we were able to achieve, under mild assumptions on the manifold $X$, a reasonably complete classification theorem. In particular, Frobenius-constant quantizations do exist.

Received by the editors October 7, 2005.

2000 Mathematics Subject Classification. Primary 14M99.

The first author was partially supported by NSF grant DMS-0071967.

The second author was partially supported by CRDF grant RM1-2694-MO05. 
Moreover, as a prerequisite to the study of quantizations, we investigate to some extent symplectic differential geometry in positive characteristic. In particular, we introduce a notion of a restricted Poisson algebra - a Poisson version of the standard notion of a restricted Lie algebra. We also prove a version of the Darboux Theorem for these algebras (Proposition 3.4; in our setting the usual Darboux Theorem is false). These results seem to be new, and they might be of independent interest: even a reader who is not interested in quantizations at all might find symplectic geometry in positive characteristic worth her (or his) attention.

Our main tool is the (version of the) technique of the so-called formal geometry; we have used the same technique in [BK1]. In positive characteristic this turns out to be simpler. In fact, we could have avoided this entirely by working systematically in the flat topology on the symplectic manifold $X$; the use of formal geometry in this context amounts to a choice of a concrete flat covering of $X$ to trivialize things. We decided to make this choice because the picture becomes more explicit and more easily accessible to people who have little experience with flat topology. Moreover, the use of formal geometry emphasizes the differential-geometric nature of the subject and the parallels with the characteristic 0 case.

Our restriction to Frobenius-constant quantizations is particularly unfortunate because - as opposed to the main result in [BK1] - it effectively excludes from consideration compact algebraic varieties, such as abelian varieties or surfaces of type K3. There undoubtedly exists a more general theory which would apply to these cases as well. Another thing which we leave out entirely in this paper is questions of mixed characteristic: a complete theory should probably incorporate both the quantizations of a characteristic $p$ manifold and its liftings to characteristic 0 . All this should be the subject of future research.

The paper is organized as follows. The first section contains the necessary definitions and statements of our main results. Unfortunately, many of this material is not standard. We have tried to keep the technical details to an absolute minimum, but Section 1 still takes about half of the paper. Since this was unavoidable anyway, we have also incorporated some of the shorter proofs into this section; eventually, Section 1 became self-contained except for four clearly marked main results - two deal with the symplectic side of the story, and the other two with quantizations. Those two that deal with things symplectic and Poisson are proved in Section 2. Then in Section 3, we restrict our attention to local study of symplectic manifolds (this includes our Darboux-type theorem for restricted Poisson algebras). Finally, in Section 4 we use the techniques of formal geometry to globalize things and to prove the remaining two results which deal with quantizations.

\section{Statements And Definitions}

In this section we give the relevant definitions and state our main results. The reader is encouraged to compare everything to [BK1, 1.3].

1.1. Definitions. Throughout the paper, we will say that a subalgebra $A \subset B$ in an algebra $B$ is central if it lies in the center of the algebra $B$, and we will say that an algebra map $A \rightarrow B$ is central if its image is central in $B$.

Fix once and for all a field $k$ of characteristic $p>2$. For any vector space, algebra, scheme, etc. $M$ over $k$ denote by $M^{(1)}=M \otimes_{k} k$ its pullback with respect to the Frobenius map Fr : $k \rightarrow k$. For a commutative algebra $A$ over $k$, denote 
by $A^{p} \subset A$ the subalgebra generated by $p$-th powers of all elements $a \in A$. By definition, the Frobenius map Fr $: A \rightarrow A$ factors through the embedding $A \subset A^{(1)}$ by means of the relative Frobenius map $\operatorname{Fr}_{k}: A^{(1)} \rightarrow A$; the subalgebra $A^{p} \subset A$ is the image of the map $\mathrm{Fr}_{k}$. If the algebra $A$ is reduced, the relative Frobenius map is injective; in this case $A^{p} \cong A^{(1)}$. As usual, by a derivation $D: A \rightarrow M$ with values in an $A$-module $M$ we will understand a $k$-linear map which satisfies the Leibnitz rule. By a Frobenius derivation $D: A \rightarrow M$ with values in an $A^{p}$-module $M$ we will understand a map which is a derivation with respect to the induced $A^{(1)}$-module structure on $M$ - in other words, we have $D(1)=0$ and

$$
D(a b)=a^{p} D(b)+b^{p} D(a) .
$$

The following definition is exactly the same as in the case char $k=0$.

Definition 1.1. By a quantization $A_{h}$ of a commutative $k$-algebra $A$ we will understand an associative flat $k[[h]]$-algebra $A_{h}$, complete with respect to the $h$-adic filtration and equipped with an isomorphism $A_{h} / h \cong A$.

By a quantization $\mathcal{O}_{h}$ of a scheme $X$ over $k$ we will understand a Zariski sheaf $\mathcal{O}_{h}$ of flat $k[[h]]$-algebras on $X$, complete with respect to the $h$-adic filtration and equipped with an isomorphism $\mathcal{O}_{h} / h \cong \mathcal{O}_{X}$.

As explained in [BK1, 1.3], quantizations of a commutative $k$-algebra $A$ are in natural one-to-one correspondence with quantizations of the affine scheme $X=$ $\operatorname{Spec} A$. Any quantization $\mathcal{O}_{h}$ of a scheme $X / k$ induces a Poisson bracket in $\mathcal{O}_{X}$; one usually keeps this structure in mind from the start and says that $\mathcal{O}_{h}$ is a quantization of the Poisson scheme $X$. We note that by definition, for any section $f \in \mathcal{O}_{h}$ which lies in the center $\mathcal{Z}_{h} \subset \mathcal{O}_{h}$ of the algebra sheaf $\mathcal{O}_{h}$, its reduction $\bar{f} \in \mathcal{O}_{X}$ modulo $h$ lies in the Poisson center $\mathcal{Z} \subset \mathcal{O}_{X}$.

Definition 1.2. A quantization $\mathcal{O}_{h}$ of a scheme $X / k$ is called central if the natural map $\mathcal{Z}_{h} \rightarrow \mathcal{Z}$ from the center $\mathcal{Z}_{h} \subset \mathcal{O}_{h}$ of the associative algebra $\mathcal{O}_{h}$ to the Poisson center $\mathcal{Z} \subset \mathcal{O}_{X}$ is surjective.

We note that the center $\mathcal{Z}_{h} \subset \mathcal{O}_{h}$ of a quantization $\mathcal{O}_{h}$ is automatically flat over $k[[h]]$ (this is equivalent to having no $h$-torsion). Thus $\mathcal{Z}_{h}$ is a commutative one-parameter deformation of some subalgebra $\mathcal{Z}_{h} / h \subset \mathcal{Z}$. The quantization is central if $\mathcal{Z}_{h} / h$ is the whole Poisson center $\mathcal{Z} \subset \mathcal{O}_{X}$.

In [BK2] we have used a notion of a Frobenius-constant quantization; roughly speaking, a quantization was called Frobenius-constant if it is central, and moreover, we have an isomorphism $\mathcal{Z}_{h} \cong \mathcal{O}_{X}^{p}[[h]]$. It turns out that for general theory, we need a more precise notion.

Lemma 1.3. Let $A$ be an associative algebra over $k$, and let $A_{(k)}, A_{(1)}=A$, $A_{(k)}=\left[A, A_{(k-1)}\right]$ be its central series with respect to the commutator. If $A_{(p)}=0$ and $A_{(2)}^{p}=0$, then setting $x \mapsto x^{p}$ defines an additive multiplicative central map $\widetilde{\mathrm{Fr}}_{k}: A^{(1)} \rightarrow A$.

Proof. Consider the tensor algebra $T^{\bullet}(A)$ generated by the vector space $A$. Since $T^{\bullet}(A)$ is the universal enveloping algebra for the free Lie algebra $L^{\bullet}(A)$, by the Poincare-Birkhoff-Witt Theorem it has an increasing filtration $F^{P B W} T^{{ }^{*}}(A)$ such that the associated graded quotient is isomorphic to the symmetric algebra $S^{\bullet}\left(L^{\bullet}(A)\right)$. Moreover, the universal enveloping algebra $T^{\bullet}(A)$ is a Hopf algebra; this 
structure is compatible with the Poincare-Birkhoff-Witt filtration, and it induces the standard Hopf algebra structure on $S^{\bullet}\left(L^{\bullet}(A)\right)$.

For any $x, y \in A$ let $d(x, y)=(x+y)^{\otimes p}-x^{\otimes p}-y^{\otimes p} \in T^{p}(A)$. The element $d(x, y)$ is primitive with respect to the Hopf algebra structure on $T^{\bullet}(A)$. A priori, it lies inside the $p$-th term $F_{p}^{P B W} T^{\bullet}(A)$ of the Poincare-Birkhoff-Witt filtration. However, the image of $d(x, y)$ in $\operatorname{gr}_{p}^{P B W} T^{p}(A) \cong S^{p}\left(L^{1}(A)\right)=S^{p}(A)$ is obviously zero, so that in fact $d(x, y) \in F_{q}^{P B W} T^{\bullet}(A)$ for some $q \leq p-1$. Since $d(x, y)$ is primitive, so is its image in $\operatorname{gr}_{q}^{P B W} T^{\bullet}(A)=S^{q}\left(L^{\bullet}(A)\right)$; since $q<p$, this is possible only if $q=1$. We conclude that $d(x, y) \in S^{1}\left(L^{p}(A)\right)=L^{p}(A)$ is a Lie polynomial in $x$ and $y$.

But since by assumption $A_{(p)}=0$, the multiplication map $m: T^{\bullet}(A) \rightarrow A$ vanishes on $L^{p}(A) \subset T^{p}(A)$. Therefore $m(d(x, y))=(x+y)^{p}-x^{p}-y^{p}=0$, and $\widetilde{\mathrm{Fr}}_{k}$ is indeed additive. Moreover, denoting ad $x(z)=[x, z]$, we have

$$
\left[x^{\otimes p}, y\right]=(\operatorname{ad} x)^{p}(y) \in L^{p+1}(A),
$$

so that $x^{p}$ is indeed central in $A$. Finally,

$$
\begin{aligned}
x^{p} y^{p} & =\frac{1}{2}\left(\left(x^{p}+y^{p}\right)^{2}-x^{2 p}-y^{2 p}\right) \\
& =\frac{1}{2}\left(\left(x^{2}+y^{2}+2 x y-[x, y]\right)^{p}-x^{2 p}-y^{2 p}\right)=(x y)^{p},
\end{aligned}
$$

which finishes the proof.

Applying this to quantizations, we see that for any quantization $A_{h}$ of a commutative algebra $A$, the quotient $A_{h} / h^{p-1}$ satisfies the conditions of the lemma.

Definition 1.4. A Frobenius-constant quantization of a $k$-algebra $A$ is a pair of a quantization $A_{h}$ of the algebra $A$ and a multiplicative central $k[h]$-linear splitting map $s: A_{h}^{(1)} \rightarrow A_{h}$ such that $s(h)=0$ and

$$
s(a)=a^{p} \quad \bmod h^{p-1}
$$

for any $a \in A_{h}$.

Assume given a Frobenius-constant quantization $A_{h}$ of a $k$-algebra $A$. Since $s(h)=0$, the splitting map $s: A_{h}^{(1)} \rightarrow A_{h}$ factors through the reduction map $A_{h}^{(1)} \rightarrow A^{(1)} \cong A_{h}^{(1)} / h$. We will say that the Frobenius-constant quantization $A_{h}$ is regular if $s$ further factors through the Frobenius map $A^{(1)} \rightarrow A^{p}$ (in other words, $s(a)=0$ for any $a \in A^{(1)}$ with $a^{p}=0$ ). If the algebra $A$ is reduced, this is automatic; in general, it might happen that for some $a \in A, a^{p}=0$ in $A$, but when we lift $a$ to an element $\widetilde{a} \in A_{h}$, the $p$-th power $\widetilde{a}^{p}$ already becomes non-trivial in $A_{h} / h^{2}$. We note that in our approach to quantizations, we really have to consider non-reduced algebras, even if the original algebra or scheme we want to quantize is smooth (see Section 3).

We also note that the notion of a regular Frobenius-constant quantization has nice functoriality properties with respect to base change. More precisely, assume given a $k$-algebra $A$, an ideal $I \subset A^{p}$, and a regular Frobenius-constant quantization $\left\langle A_{h}, s\right\rangle$ of $A$. Then the quotient $A_{h} / s(I) A_{h}$ is a regular Frobenius-constant quantization of the quotient $A / I A$. 
1.2. Universal polynomials. To proceed further, we need to develop some formalism on the $p$-th power operation in characteristic $p$. It will be convenient to interpolate between the notions of an associative and a Poisson algebra by introducing the following definition.

Definition 1.5. A quantized algebra $A$ over a field $k$ is an associative algebra over the algebra $k[[h]]$ of formal power series in one variable $h$ equipped with an additional $k[h]$-linear Lie bracket $\{-,-\}$, which is a derivation in each variable and satisfies

$$
h\{a, b\}=a b-b a
$$

for every $a, b \in A$.

A quantized algebra which is flat over $k[[h]]$ - equivalently, has no $h$-torsion is the same as a flat associative $k[[h]]$-algebra $A$ with commutative quotient $A / h A$ (the bracket $\{a, b\}$ is uniquely defined by (1.2)). On the other hand, a quantized algebra annihilated by $h$ is the same as a Poisson algebra. If $V$ is a finite-dimensional vector space over $k$, then the free associative algebra over $k$ generated by $V$ is the tensor algebra $T^{\bullet} V$. Since $T^{\bullet}(V)$ is also the universal enveloping algebra of the free Lie algebra $L^{\bullet}(V)$ generated by $V$, it has a Poincare-Birkhoff-Witt filtration $F^{P B W} T^{\bullet} V$, and the free Poisson algebra $P^{\bullet} V$ is the associated graded $\operatorname{gr}^{P B W} T^{\bullet} V$ with respect to the PBW filtration. As for the free quantized algebra $Q^{\bullet} V$, it is the so-called Reese algebra associated to the PBW filtration: we have

$$
Q^{\bullet} V=\bigoplus F \cdot T^{\bullet} V
$$

and the variable $h$ acts as the natural embedding $h: F \cdot T^{\bullet} V \rightarrow F_{\bullet+1} T^{\bullet} V$. When $V=k\left\langle x_{0}, \ldots, x_{n}\right\rangle$ is spanned by basis elements $x_{0}, \ldots, x_{n}$, we will denote the algebra $Q^{\bullet} V$ by $Q^{\bullet}\left(x_{0}, \ldots, x_{n}\right)$ and call its elements quantized polynomials in variables $x_{0}, \ldots, x_{n}$.

Now consider the algebra $Q^{\bullet}(x, y)$ of quantized polynomials in two variables $x, y$. On one hand, this algebra has no $h$-torsion; on the other hand, the quotient $Q^{\bullet}(x, y) / h^{p-1}$ obviously satisfies the assumptions of Lemma 1.3. We conclude that there exist two canonical quantized polynomials $L(x, y), P(x, y)$ such that

$$
\begin{aligned}
& h^{p-1} L(x, y)=(x+y)^{p}-x^{p}-y^{p}, \\
& h^{p-1} P(x, y)=(x y)^{p}-x^{p} y^{p} .
\end{aligned}
$$

As noted in the proof of Lemma 1.3, the polynomial $L(x, y)$ is in fact a Lie polynomial in $x, y$. It is well-known, and it can be computed explicitly (the Jacobson formula; see, e.g., [DP, II, $\S 7.3$, Définition 3.1]). We will only need an easy "leading term" formula; for the convenience of the reader, we include a proof.

Lemma 1.6. Assume given a Lie algebra $L$ equipped with a two-step decreasing filtration, $F^{1} L \subset F^{0} L=L,\left[F^{i} L, F^{j} L\right] \subset F^{i+j} L$ for any $i, j$. Then for any $x \in L$, $y \in F^{1} L$ we have

$$
L(x, y)=(\operatorname{ad} x)^{p-1}(y) .
$$

Proof. Since ad $x$ preserves $F^{1} L$ and $F^{2} L=0$, so that the bracket on $F^{1} L$ vanishes, the only non-trivial Lie monomial in $x$ and $y$ of arbitrary degree $l$ is $(\operatorname{ad} x)^{l}(y)$ (the proof is an obvious induction on $l)$. Therefore $L(x, y)=\lambda(\operatorname{ad} x)^{p-1}(y)$ for some 
universal constant $\lambda \in \mathbb{Z} / p \mathbb{Z}$. Now consider the Lie algebra $L$ spanned by $x$ and $y$, with $F^{1} L \subset L$ spanned by $y$, and $\{x, y\}=y$. Then we have

$$
\begin{aligned}
\lambda y & =\lambda(\operatorname{ad} x)^{p}(y)=(\operatorname{ad} x)\left(\lambda(\operatorname{ad} x)^{p-1}(y)\right)=(\operatorname{ad} x)(L(x, y)) \\
& =(\operatorname{ad}(x+y))^{p}(x)-(\operatorname{ad} x)^{p}(x)-(\operatorname{ad} y)^{p}(x)=y,
\end{aligned}
$$

which proves that $\lambda=1$.

The Lie polynomial $L(x, y)$ is used to define the standard notion of a so-called restricted Lie algebra structure in characteristic $p$.

Definition 1.7. A restricted Lie algebra $A$ over a field of characteristic $p$ is a Lie algebra $A$ over $k$ equipped with an additional operation $x \mapsto x^{[p]}$ such that $(a x)^{[p]}=a^{p} x^{[p]}$ for any $a \in k, x \in A$, and

$$
\begin{aligned}
\left\{x^{[p]}, y\right\} & =(\operatorname{ad} x)^{p}(y), \\
(x+y)^{[p]} & =x^{[p]}+y^{[p]}+L(x, y)
\end{aligned}
$$

for any $x, y \in A$, where we denote ad $x(z)=\{x, z\}$.

We will need a Poisson version of this notion.

Definition 1.8. A restricted Poisson algebra $A$ over a field $k$ of characteristic $p$ is a Poisson algebra $A$ over $k$ equipped with an operation $x \mapsto x^{[p]}$, which turns $\langle A,\{-,-\}\rangle$ into a restricted Lie algebra and satisfies

$$
(x y)^{[p]}=x^{p} y^{[p]}+x^{[p]} y^{p}+P(x, y)
$$

for any $x, y \in A$.

More generally, here is a version for arbitrary quantized algebras.

Definition 1.9. A restricted structure on a quantized algebra $A$ over $k$ is given by an operation $x \mapsto x^{[p]}$ on $A$ which turns $\langle A,\{-,-\}\rangle$ into a restricted Lie algebra, preserves $h$ - that is, $h^{[p]}=h-$ and satisfies

$$
(x y)^{[p]}=x^{p} y^{[p]}+x^{[p]} y^{p}-h^{p-1} x^{[p]} y^{[p]}+P(x, y)
$$

for any $x, y \in A$.

As an immediate corollary of this definition, we see that for any element $x \in A$ of a restricted quantized algebra $A$ we have

$$
(h x)^{[p]}=h x^{p} .
$$

If $A$ is a Poisson algebra, we have $h A=0$, so that these two definitions agree. If the Poisson bracket on $A$ is trivial, then the $p$-th power operation is given by a Frobenius derivation $K: A \rightarrow A$; thus a restricted Poisson algebra with trivial bracket is the same as a commutative algebra $A$ equipped with a Frobenius derivation $K: A \rightarrow$ $A$. Even if the bracket on $A$ is not trivial, the difference between two restricted structures is a Frobenius derivation of $A$ (moreover, by (1.4) it must take values in the Poisson center of $A$ ). In general, examples of restricted quantized algebras are provided by Frobenius-constant quantizations. Namely, assume given a Frobeniusconstant quantization $A_{h}$ of a commutative algebra $A$ over $k$, with splitting map $s: A_{h}^{(1)} \rightarrow A_{h}$. Then for any integer $n$, the quotient $A_{n}=\widetilde{A} / h^{n+1}$ is a quantized algebra, and this algebra is restricted: a natural $p$-th power operation is given by

$$
x^{[p]}=\frac{1}{h^{p-1}}\left(x^{p}-s(x)\right) .
$$


Checking the conditions of Definition 1.9 is straightforward and left to the reader. Conversely, every restricted quantized algebra $A_{h}$ without $h$-torsion is a Frobeniusconstant quantization of its quotient $A=A_{h} / h$ : using (1.4), (1.5), and (1.7), one easily checks that $s(x)=x^{p}-h^{p-1} x^{[p]}$ gives a central splitting map $s: A_{h}^{(1)} \rightarrow A_{h}$ which vanishes on $h A_{h}^{(1)} \subset A_{h}^{(1)}$.

1.3. Restricted structures. We can now state our results. We fix the base field $k$ of odd positive characteristic, and we only consider algebras and schemes over $k$. We will say that a Noetherian scheme $X$ equipped with a map $\pi: X \rightarrow S$ to a Noetherian scheme $S$ is quasiregular over $S$ if $\pi$ is flat, $X$ is of finite type over $S$, and the sheaf $\Omega^{1}(X / S)$ of relative Kähler differentials is flat over $\mathcal{O}_{X}$. A scheme $X$ is quasiregular if it is quasiregular over the point Spec $k$. A regular scheme $X$ of finite type is of course quasiregular; since $p=$ char $k$ is positive, the converse is true only if $X$ is reduced (and at least one non-reduced quasiregular algebra will be really important to us; see Section 3 below). Most of the usual facts of differential calculus works for quasiregular schemes without any changes - in particular, we have flat sheaves $\Omega^{\bullet}(X / S)$ of relative differential forms, a flat relative tangent bundle $\mathcal{T}(X / S)$, the de Rham differential on $\Omega^{\bullet}(X / S)$, and the Cartan homotopy formula describing the natural action of the Lie algebra $\mathcal{T}(X / S)$ on $\Omega^{\bullet}(X / S)$.

It is customary to consider the pullback $X \times_{\mathrm{Fr}_{S}} S$ of $X / S$ with respect to the Frobenius map $\mathrm{Fr}_{S}: S \rightarrow S$, and to factor the Frobenius map $\operatorname{Fr}_{X}: X \rightarrow X$ through the pullback map id $\times \mathrm{Fr}_{S}: X \times_{\mathrm{Fr}_{S}} S \rightarrow X$ by the relative Frobenius map $\operatorname{Fr}_{X / S}: X \rightarrow X \times_{\mathrm{Fr}_{S}} S$. In other words, one considers the commutative diagram

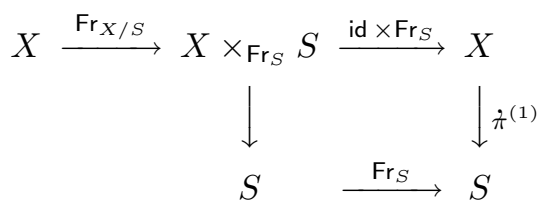

where the square on the right is Cartesian. However, it will be more convenient for us to consider the sheaf $\mathcal{O}_{X}^{[p]}=\mathcal{O}_{X}^{p} \otimes_{\mathcal{O}_{S}^{p}} \mathcal{O}_{S}$ of algebras on $X$ and the scheme $X^{[p]}=\left\langle X, \mathcal{O}_{X}^{[p]}\right\rangle$. If $X$ and $S$ are reduced, we have $X^{[p]} \cong X \times_{\mathrm{Fr}_{S}} S$. If if they are not reduced, the scheme $X^{[p]}$ is still quasiregular, and the Frobenius map $\operatorname{Fr}_{X}: X \rightarrow X$ still factors through a map $\operatorname{Fr}_{X}[p]: X^{[p]} \rightarrow X$ by means of a map which we will, by abuse of notation, denote by $\operatorname{Fr}_{X / S}: X \rightarrow X^{[p]}$.

One easily checks that the sheaf $\mathcal{O}_{X}^{[p]}$ of functions on $X^{[p]}$ coincides with the subsheaf $\mathcal{O}_{X, c l} \subset \mathcal{O}_{X}$ of functions closed with respect to the relative de Rham differential. Therefore the de Rham differential on $X / S$ is $\mathcal{O}_{X}^{[p]}$-linear, so that the de Rham complex $\operatorname{Fr}_{X / S *} \Omega^{\bullet}(X / S)$ is a complex of coherent sheaves of $\mathcal{O}_{X}^{[p]}$-modules on the scheme $X^{[p]}$. In particular, we have the coherent $\mathcal{O}_{X}^{[p]}$-module subsheaves $\operatorname{Fr}_{X / S *} \Omega_{c l}^{\bullet}(X / S) \subset \operatorname{Fr}_{X / S *} \Omega^{\bullet}(X / S)$ of closed forms.

The Zariski cohomology sheaves $\mathcal{H}^{\bullet}\left(\operatorname{Fr}_{X / S *} \Omega^{\bullet}(X / S)\right)$ are identified with the sheaves $\Omega_{[p]}^{\bullet}(X / S)=\operatorname{Fr}_{X[p]}^{*} \Omega^{\bullet}(X / S)$ by the canonical $\mathcal{O}_{X}^{[p]}$-linear Cartier operations $C^{\bullet}: \operatorname{Fr}_{X / S *} \Omega_{c l}^{\bullet}(X / S) \rightarrow \Omega_{[p]}^{\bullet}(X / S)$ (this generalizes the isomorphism $\mathcal{O}_{X, c l} \cong$ $\left.\mathcal{O}_{X}^{[p]}\right)$. The canonical filtration on $\Omega^{\bullet}(X / S)$ induces an increasing filtration $F$. on 
the relative de Rham cohomology sheaves $\mathbf{R}^{\bullet} \pi_{*}\left(\Omega^{\bullet}(X / S)\right.$ known as the conjugate filtration; the Cartier operations $C^{\bullet}$ give the projections $F_{j} \mathbf{R}^{k} \pi_{*}\left(\Omega^{\bullet}(X / S)\right) \rightarrow$ $\mathbf{R}^{k-j} \pi_{*} \Omega_{[p]}^{j}(X / S)$ onto the associated graded pieces of this filtration.

We also apply the standard deformation theory to the family $X / S$; thus for any derivation $K: \mathcal{O}_{S} \rightarrow \mathcal{O}_{S}$, we have the Kodaira-Spencer deformation class $\theta_{K} \in$ $H^{1}(X, \mathcal{T}(X / S))$. This class is represented by an explicit $\mathcal{T}(X / S)$-torsor $\left[\theta_{K}\right]$ over $X$ : local sections of $\left[\theta_{K}\right]$ are derivations of the structure sheaf $\mathcal{O}_{X}$ whose restrictions to $\pi^{-1} \mathcal{O}_{S} \subset \mathcal{O}_{X}$ coincide with $K$. For any relative $k$-form $\alpha \in H^{0}\left(X, \Omega^{k}(X / S)\right)$, contraction with $\alpha$ gives a sheaf map $\left.\mathcal{T}(X / S) \rightarrow \Omega^{k-1}(X / S), \xi \mapsto \alpha\right\lrcorner \xi$. By means of this map, the torsor $\left[\theta_{K}\right]$ induces an $\Omega^{k-1}(X / S)$-torsor on $X$ which we will denote by $\alpha\lrcorner\left[\theta_{K}\right]$. This immediately generalizes to derivations $K$ with values in some sheaf of $\mathcal{O}_{S}$-modules; in particular, for any Frobenius derivation $K: \mathcal{O}_{S} \rightarrow \mathcal{O}_{S}$ and any form $\alpha \in \Omega^{k}(X / S)$, we obtain an $\Omega_{[p]}^{k-1}(X / S)$-torsor $\left.\operatorname{Fr}_{X[p]}^{*} \alpha\right\lrcorner\left[\theta_{K}\right]$.

Finally, for any vector field $\xi$ on $X$, the $p$-th power of the corresponding derivation of the structure sheaf $\mathcal{O}_{X}$ is also a derivation; therefore the Lie algebra $\mathcal{T}(X / S)$ of relative vector fields on $X$ carries a natural restricted Lie algebra structure.

By a symplectic manifold over $S$ we will understand a Noetherian quasiregular scheme $X / S$ equipped with a non-degenerate closed relative 2 -form

$$
\Omega \in H^{0}\left(X, \Omega^{2}(X / S)\right) .
$$

Just as in the case of characteristic 0 , such a manifold is automatically equipped with an $\mathcal{O}_{S}$-linear Poisson bracket. For any local function $f \in \mathcal{O}_{X}$ we denote the derivation $g \mapsto\{f, g\}$ of the sheaf $\mathcal{O}_{X}$ by $H_{f}$; explicitly, we have $\left.H_{f}\right\lrcorner \Omega=d f$. Vector fields $H_{f}$ are called Hamiltonian and form a subsheaf of Lie subalgebras $H(X) \subset \mathcal{T}(X / S)$ in the relative tangent sheaf $\mathcal{T}(X / S)$.

Lemma 1.10. Assume given a symplectic manifold $X / S$. Then the Poisson center $\mathcal{Z} \subset \mathcal{O}_{X}$ coincides with the subsheaf $\mathcal{O}_{X}^{[p]} \subset \mathcal{O}_{X}$.

Proof. A function $f \in \mathcal{O}_{X}$ lies in the Poisson center if and only if $H_{f}=0$. But since $\Omega$ is non-degenerate, this is equivalent to $\left.H_{f}\right\lrcorner \Omega=0$, which means $d f=0$.

As a corollary of this lemma, we see that for symplectic manifolds over $k$, a quantization $\mathcal{O}_{h}$ is Frobenius-constant if and only if (a) it is central, and (b) the center $\mathcal{Z}_{h}$ is isomorphic to $\mathcal{O}_{X}^{p}[[h]]$ - indeed, any splitting map $\mathcal{O}_{X}^{p} \cong \mathcal{Z} \rightarrow \mathcal{Z}_{h}$ induces an isomorphism $\mathcal{O}_{X}^{p}[[h]] \cong \mathcal{Z}_{h}$ and conversely, any map $\mathcal{O}_{X}^{p} \rightarrow \mathcal{O}_{X}^{p}[h] / h^{p-1} \cong \mathcal{Z}_{h} / h^{p-1}$ can be obviously lifted to a map $\mathcal{O}_{X}^{p} \rightarrow \mathcal{O}_{X}^{p}[[h]]$. Therefore - at least in the case $S=$ Spec $k$ - our Definition 1.4 and [BK2, Definition 3.3] impose equivalent conditions on quantizations. The difference is in the additional structure (not every splitting map in [BK2, Definition 3.3] would serve for Definition 1.4).

Our first general result in this paper is the following.

Theorem 1.11. Assume given a symplectic manifold $X / S$. Then the following conditions are equivalent:

(i) The subalgebra of Hamiltonian vector fields in $\mathcal{T}(X / S)$ is closed with respect to the restricted Lie algebra operation $\xi \mapsto \xi^{[p]}$.

(ii) We have $C^{2}(\Omega)=0 \in H^{0}\left(X, \Omega_{[p]}^{2}(X / S)\right)$.

Moreover, if $X$ admits a central quantization $\mathcal{O}_{h}$, then both these conditions are satisfied. 
The proof of this theorem is given in Subsection 2.1 (page 425). As an immediate corollary, we see that if a symplectic manifold $X$ admits a central quantization, or at least satisfies condition (i) of Theorem 1.11, then the de Rham cohomology class $[\Omega] \in H_{D R}^{2}(X / S)$ lies in the first term $F_{1} H_{D R}^{2}(X / S)$ of the conjugate filtration. In fact, $[\Omega]$ comes from a cohomology class

$$
\overline{[\Omega]} \in H^{1}\left(X, \Omega_{c l}^{1}(X / S)\right) .
$$

Indeed, geometrically Theorem 1.11 (i) means that the form $\Omega$ is locally exact in Zariski topology. The 1-forms $\alpha$ such that $d \alpha=\Omega$ form a torsor $\mathcal{M}_{\Omega}$ over the vector bundle $\operatorname{Fr}_{X / S *} \Omega_{c l}^{1}(X / S)$ on $X^{[p]}$ spanned by closed 1-forms on $X$; this torsor represents the class $\overline{[\Omega]}$. Applying the Cartier map $C^{1}: \operatorname{Fr}_{X / S *} \Omega_{c l}^{1}(X / S) \rightarrow$ $\Omega_{[p]}^{1}(X / S)$, we can also consider the induced $\Omega_{[p]}^{1}(X / S)$-torsor $C_{*}^{1} \mathcal{M}_{\Omega}$.

Assume now that the base scheme $S$ is equipped with a Frobenius derivation $K: \mathcal{O}_{S} \rightarrow \mathcal{O}_{S}$, so that $\mathcal{O}_{S}$ becomes a restricted Poisson algebra with trivial Poisson bracket. By a restricted structure on the Poisson scheme $X / S$ we will understand a restricted structure on the Poisson algebra sheaf $\mathcal{O}_{X}$ such that the embedding $\pi^{-1} \mathcal{O}_{S} \rightarrow \mathcal{O}_{X}$ is a (central) restricted Poisson algebra map. Then we have the following (the proof is in Subsection 2.3, on page 427).

Theorem 1.12. Let $X$ be a symplectic manifold over $S$ which satisfies the equivalent conditions of Theorem 1.11. Then restricted structures on the Poisson scheme $X / S$ are in natural one-to-one correspondence with isomorphisms

$$
\left.C_{*}^{1} \mathcal{M}_{\Omega} \cong \operatorname{Fr}_{X[p]}^{*} \Omega\right\lrcorner\left[\theta_{K}\right]
$$

of torsors over the bundle $\Omega_{[p]}^{1}(X / S)$.

This theorem immediately gives a necessary condition for the existence of Frobenius-constant quantizations. Namely, assume given a symplectic manifold $X / S$, $\pi: X \rightarrow S$, and a Frobenius derivation $K: \mathcal{O}_{S} \rightarrow \mathcal{O}_{S}$. We will say that a regular Frobenius-constant quantization $\left\langle\mathcal{O}_{h}, s\right\rangle$ of the Poisson scheme $X$ is compatible with the derivation $K$ if for any local section $f \in H^{0}\left(U, \mathcal{O}_{h}\right)$ whose reduction $\bar{f} \in H^{0}\left(U, \mathcal{O}_{X}\right)$ modulo $h$ comes from a function $b \in H^{0}\left(S, \mathcal{O}_{S}\right)$ on $S, \bar{f}=\pi^{*}(b)$, we have

$$
f^{p}-s\left(f^{p}\right)=\pi^{*}(K(b)) .
$$

Corollary 1.13. Assume given a symplectic manifold $X / S$, a Frobenius derivation $K: \mathcal{O}_{S} \rightarrow \mathcal{O}_{S}$, and a regular Frobenius-constant quantization $\left\langle\mathcal{O}_{h}, s\right\rangle$ of the Poisson scheme $X$ compatible with $K$. Then the cohomology class $[\Omega] \in H_{D R}^{2}(X / S)$ of the symplectic form $\Omega$ lies in the first term $F_{1} H_{D R}^{2}(X / S)$ of the conjugate filtration, and satisfies

$$
\left.C^{1}([\Omega])=\left(\operatorname{Fr}_{X[p]}^{*}[\Omega]\right)\right\lrcorner \theta_{K} \in H^{1}\left(X, \Omega_{[p]}^{1}(X / S)\right) .
$$

In particular, if $K=0$ (for instance, if $S=\operatorname{Spec} k$ ), then the class $[\Omega]$ lies in the second term $F_{2} H_{D R}^{2}(X / S)$ of the conjugate filtration.

Proof. As we have noted in the definition of the restricted quantized algebras, setting

$$
x^{[p]}=x^{p}-\frac{1}{h^{p-1}} s(x)
$$

gives a restricted quantized algebra structure on the sheaf $\mathcal{O}_{h}$. Reducing modulo $h$, we obtain a restricted Poisson structure on the quotient $\mathcal{O}_{h} / h \cong \mathcal{O}_{X}$. Compatibility 
condition (1.8) ensures that this structure is compatible with the restricted Poisson structure on $\mathcal{O}_{S}$ given by $K$. We can now apply Theorem 1.11 (i) and Theorem 1.12, and the only thing that remains to be proved is that the quantization $\mathcal{O}_{h}$ is central. Indeed, the splitting map $s$ induces a map $s: \mathcal{O}_{X}^{p} \otimes_{B^{p}} B_{h} \rightarrow \mathcal{Z}_{h}$, which reduces to $\mathcal{O}_{X}^{p} \otimes_{B^{p}} B \rightarrow \mathcal{Z}_{h} / h \subset \mathcal{Z}$ modulo $h$. But by Lemma 1.10 the composition $\mathcal{O}_{X}^{p} \otimes_{B^{p}} B \cong \mathcal{O}_{X / S}^{p} \rightarrow \mathcal{Z}$ is an isomorphism. Therefore $\mathcal{Z}_{h} / h \cong \mathcal{Z}$.

Remark 1.14. The conditions $C([\Omega])=0$ and furthermore $C^{1}([\Omega])=0$ are not very natural for projective symplectic manifolds such as surfaces of type $K 3$ and abelian varieties of even dimension. At least for abelian varieties, a more natural condition is $C([\Omega])=[\Omega]$. It would be very interesting to develop a parallel theory for symplectic manifolds of this type. We do not know what should replace the condition $C^{1}([\Omega])=0$.

1.4. Quantizations. We now turn to existence results for quantizations. We change our point of view: we assume from now on that the base scheme $S$ is local, we replace $X / S$ with its special fiber, and we incorporate both quantizations and the family $X / S$ into a single multi-parameter partially non-commutative deformation of the structure sheaf. To package the data, we introduce the following.

Definition 1.15. A quantization base is a commutative Noetherian complete local $k[[h]]$-algebra $B$ with maximal ideal $\mathfrak{m}_{B} \subset B, h \in \mathfrak{m}_{B}, k \cong B / \mathfrak{m}_{B}$ equipped with a map $K: B \rightarrow B^{(1)}$ such that setting $b^{[p]}=K(b)$ for any $b \in B$ turns $B$ into a restricted quantized algebra (with trivial bracket).

Assume given a symplectic manifold $X / k$ and a quantization base $B$ with maximal ideal $\mathfrak{m}_{B} \subset B$. By a $B$-quantization $\mathcal{O}^{B}$ of the manifold $X$ we will understand a sheaf $\mathcal{O}^{B}$ of restricted quantized flat $B$-algebras on $X$ equipped with a restricted Poisson isomorphism isomorphism $\mathcal{O}^{B} / \mathfrak{m}_{B} \mathcal{O}^{B} \cong \mathcal{O}_{X}$. In other words, $\mathcal{O}^{B}$ is equipped with a central restricted quantized map $B \rightarrow H^{0}\left(X, \mathcal{O}^{B}\right), \mathcal{O}^{B}$ with the induced $B$-module structure is flat over $B$, and the quotient $\mathcal{O}^{B} / \mathfrak{m}_{B} \mathcal{O}^{B}$ is identified with $\mathcal{O}_{X}$. In the case $B=k[[h]]$, this reduces to Definition 1.4, so that a $k[[h]]$-quantization is the same as a Frobenius-constant quantization. Another basic example is $B=k$; a $k$-quantization is the same as a restricted structure on the Poisson scheme $X$. We will say that a $B$-quantization $\mathcal{O}^{B}$ is regular if for any local section $f \in H^{0}\left(U, \mathcal{O}^{B}\right)$ whose reduction $\bar{f} \in H^{0}\left(U, \mathcal{O}_{X}\right) \bmod \mathfrak{m}_{B}$ satisfies $\bar{f}^{p}=0$, we have $f^{p}=h^{p-1} f^{[p]}$. As before, this condition is automatic if $X / k$ is reduced.

We construct quantizations by induction, going step-by-step through a sequence of extensions of the quantization base. To make this precise, we say that by an extension $\langle B, I \subset B\rangle$ of a quantization base $B_{0}$ we will understand a quantization base $B$ equipped with a (restricted) ideal $I \subset B$ and an isomorphism $B / I \cong B_{0}$. We will say that an extension $\langle B, I\rangle$ is small if the ideal $I \subset B$ is annihilated by $\mathfrak{m}_{B}$. For example, for any quantization base $B$ and any integer $n$, the quotient $B / \mathfrak{m}_{B}^{n+1}$ is a small extension of the quotient $B / \mathfrak{m}_{B}^{n}$.

Our main result gives the induction step - we describe in detail how to prolong a quantization over a small extension of its base. Namely, by a small Dieudonné module over $k$ we will understand a vector space $I$ equipped with a $p$-linear map $K_{I}: I \rightarrow I, K_{I}(a v)=a^{p} K_{I}(v)$ for any $a \in k, v \in I$ (for a reader familiar with the notion of a Dieudonné module, we note that "small" here means that the map 
$V$ is trivial, $V=0$, and we take $F=K)$. We note that for any small extension $\langle B, I\rangle$, the ideal $I$ carries a natural structure of a small Dieudonné module: we set $K_{I}(x)=x^{[p]}$.

Definition 1.16. Let $X / k$ be a symplectic manifold, and assume given a small Dieudonné module $I$ over $k$. By

$$
\mathcal{H}\langle I\rangle \subset \mathrm{Fr}_{*} \Omega_{c l}^{1} \otimes_{k} I
$$

we will denote the sheaf of closed $I$-valued 1-forms $\alpha$ on $X$ satisfying $C^{1}(\alpha)=$ $K_{I}(\alpha)$.

Remark 1.17. We note that unless $K_{I}=0$, the sheaf $\mathcal{H}\langle I\rangle$ is not a sheaf of $\mathcal{O}_{X}^{[p]}$ modules. Indeed, the Cartier map $C^{1}$ is $\mathcal{O}_{X}^{[p]}$-linear, but the map $K_{I}$ is not: we have $K_{I}(f \alpha)=f^{p} K_{I}(\alpha)$.

Proposition 1.18. Assume given a small extension $\langle B, I\rangle$ of a quantization base $B_{0}$ and a regular $B_{0}$-quantization $\mathcal{O}_{h}$ of a symplectic manifold $X / k$. Denote by $Q\left(B, \mathcal{O}_{h}\right)$ the set of isomorphism classes of all $B$-quantizations $\mathcal{O}_{h}^{\prime}$ of $X / k$ equipped with an isomorphism $\mathcal{O}_{h}^{\prime} / I \cong \mathcal{O}_{h}$. Then the set $Q\left(B, \mathcal{O}_{h}\right)$ is described by the étale cohomology of the sheaf $\mathcal{H}\langle I\rangle$ on $X$ in the following way:

(i) There exists a canonical obstruction class $c\left(\mathcal{O}_{h}\right) \in H_{\text {et }}^{2}(X, \mathcal{H}\langle I\rangle)$ such that $Q\left(B, \mathcal{O}_{h}\right)$ is non-empty if and only if $c\left(\mathcal{O}_{h}\right)=0$.

(ii) If the set $Q\left(B, \mathcal{O}_{h}\right)$ is not empty, then it has a natural structure of a torsor over the group $H_{\text {et }}^{1}(X, \mathcal{H}\langle I\rangle)$.

This proposition is proved in Subsection 4.2 on page 436. As usual, one can give a more precise result which also describes the category of the quantizations $\mathcal{O}_{h}^{\prime}$ and its gerb structure; we do not do this to save space.

Our second result is somewhat surprising.

Proposition 1.19. In the assumptions of Proposition 1.18, assume in addition that $B_{0}=k$, so that $I=\mathfrak{m}_{B}$ and $B$ is an arbitrary quantization base with $\mathfrak{m}_{B}^{2}=0$. Also assume given a restricted structure on the Poisson scheme $X / k$, so that $\mathcal{O}_{X}$ becomes a $k$-quantization. Then the obstruction class $c\left(\mathcal{O}_{X}\right)$ tautologically vanishes, and the torsor $Q\left(B, \mathcal{O}_{X}\right)$ is trivial: we have a natural identification

$$
Q\left(B, \mathcal{O}_{X}\right) \cong H^{1}\left(X, \mathcal{H}\left\langle\mathfrak{m}_{B}\right\rangle\right) .
$$

The proof is also in Subsection 4.2, on page 436. To get a feeling for what Proposition 1.18 and Proposition 1.19 mean in practice, we need to get a handle on the sheaves $\mathcal{H}\langle V\rangle$. Specifically, we need one particular case: $V=k$ is onedimensional, and $K: V \rightarrow V$ is given by $K(a)=a^{p} \lambda$ for some constant $\lambda \in k$. We denote this Dieudonné module by $k\langle\lambda\rangle$, and we denote the corresponding sheaf by $\mathcal{H}\langle\lambda\rangle$. If $\lambda=0$, then $\mathcal{H}=\mathcal{H}\langle 0\rangle$ is the sheaf of closed 1-forms $\alpha$ on $X$ with $C^{1}(\alpha)=0$ (in other words, the sheaf of exact 1 -forms). Thus we have a short exact sequence

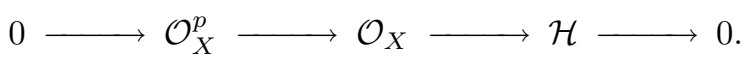

The right-hand side map is the de Rham differential, and the left-hand side map is the Frobenius map on $X$. If $\lambda=1$, then $\mathcal{H}_{\text {log }}=\mathcal{H}\langle 1\rangle$ is the sheaf of closed 1-forms with $C^{1}(\alpha)=\alpha$. It is well-known that these are of the form $\alpha=d f / f$, where $f$ is an invertible function on $X$. Thus we have a short exact sequence

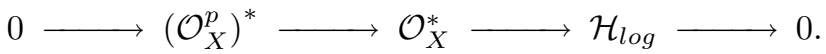


This is a version of the Kummer exact sequence. The map on the right-hand side is the $d \log$-map, $f \mapsto d f / f$, and the map on the left-hand side is again the Frobenius map, which for the multiplicative group $\mathcal{O}_{X}^{*}$ means just the multiplication by $p$.

Remark 1.20. Both (1.10) and (1.11) are examples of a more general construction. The category of small Dieudonné modules is equivalent to the category of algebraic groups over $k$ annihilated by $p$ (see, e.g., [D]). Algebraic groups over $k$ are the same as group sheaves on Spec $k$ in flat topology; by pullback, an algebraic group $G$ defines a sheaf $\mathcal{G}$ on $X$ in flat topology. If we denote by $\rho$ the natural map from $X$ with flat topology to $X$ with étale topology, then $\mathbf{R}^{i} \rho_{*} \mathcal{G}=0$ unless $i=1$. Moreover, if $G=G_{I}$ corresponds to a Dieudonné module $V$, then $\mathbf{R}^{1} \rho_{*} \mathcal{G} \cong \mathcal{H}\langle I\rangle$. The sequence (1.10) computes this higher direct image for $k\langle 0\rangle$ (which corresponds to the group of points of order $p$ in $\mathbb{G}_{a}$ ), while (1.11) corresponds to $k\langle 1\rangle$ (the group of points of order $p$ in $\left.\mathbb{G}_{m}\right)$. The reader can find more details, for instance, in $[\mathrm{M}$, III, §4].

Unfortunately, we cannot say anything about the obstruction class $c\left(\mathcal{O}_{h}\right)$ in Proposition 1.18 (i); to get results, we have to kill it off by imposing a condition on the symplectic manifold $X$.

Definition 1.21. A scheme $X$ is called admissible if it is reduced, and the Frobenius map $\mathrm{Fr}: H^{i}\left(X^{[p]}, \mathcal{O}_{X}^{[p]}\right) \rightarrow H^{i}\left(X, \mathcal{O}_{X}\right)$ is bijective for $i=1,2,3$.

By the cohomology long exact sequence associated to (1.10), we see that an admissible scheme $X$ satisfies

$$
H^{1}(X, \mathcal{H}) \cong H^{2}(X, \mathcal{H})=0 .
$$

The sheaf $\mathcal{H}$ is in fact a coherent sheaf of $\mathcal{O}_{X}^{p}$-modules, so its cohomology is the same in all topologies one might want to use, including the Zariski topology. We note that the admissibility condition always holds if $X$ is reduced, projective and ordinary over $S=$ Spec $k$, or if for some reason $H^{i}\left(X, \mathcal{O}_{X}\right)=0$ for $i=1,2,3$ (by the Grauert-Riemenschneider Theorem, this is always the case when $X$ is projective over an affine $Y$ and lifts to a smooth manifold over the ring $W_{2}(k)$ of second Witt vectors of the field $k$ ).

We can now deduce existence results for quantizations of admissible symplectic manifolds.

Proposition 1.22. Let $X / k$ be an admissible symplectic manifold equipped with a restricted structure, and let $B$ be a quantization base with maximal ideal $\mathfrak{m}_{B} \subset$ $B$. Then the set $Q(B, X)$ of isomorphism classes of regular $B$-quantizations $\mathcal{O}^{B}$ of $X$ equipped with a restricted Poisson isomorphism $\mathcal{O}^{B} / \mathfrak{m}_{B} \cong \mathcal{O}_{X}$ is naturally identified with the cohomology group $H_{\text {et }}^{1}\left(X, \mathcal{H}\left\langle\mathfrak{m}_{B} / \mathfrak{m}_{B}^{2}\right\rangle\right)$.

Proof. Filter the local algebra $B$ by powers of the maximal ideal $\mathfrak{m}_{B}$, and denote $B_{n}=B / \mathfrak{m}_{B}^{n+1}$. Then $B_{n+1}$ is a small extension of $B_{n}$, with ideal $I=\mathfrak{m}_{B}^{n+1} / \mathfrak{m}_{B}^{n+2}$. However, it immediately follows from Definition 1.9 that whenever $n \geq 1$, for every $b \in \mathfrak{m}_{B}^{n+1} \subset B$ we have $b^{[p]} \in \mathfrak{m}_{B}^{n+2} \subset B$. Therefore the map $K: I \rightarrow I$ given by the restricted structure is trivial for $n \geq 1$. By Proposition 1.18, we conclude that every regular $B_{1}$-quantization of $X$ extends uniquely to a $B$-quantization. It remains to note that $B_{1}=B / \mathfrak{m}_{B}^{2}$ satisfies the assumptions of Proposition 1.19.

Theorem 1.23. Assume given a quantization base $B$ and a symplectic manifold $X / S$ over $S=\operatorname{Spec}(B / h B)$ such that the special fiber $X_{o}=X / \mathfrak{m}_{B}$ is admissible. 
Also assume given a restricted structure on the Poisson scheme $X / S$ compatible with the natural restricted structure on $B / h B$. Then this restricted structure extends to a regular Frobenius-constant quantization of $X$ compatible with the restricted structure on $B$ as in (1.8). Moreover, the set $Q\left(B, \mathcal{O}_{X}\right)$ of isomorphism classes of all such quantizations is naturally a torsor over the group $H_{\text {et }}^{1}\left(X, \mathcal{H}_{\text {log }}\right)$.

Proof. Consider the sets $Q\left(B, X_{o}\right), Q\left((B / h B), X_{o}\right)$ of isomorphism classes of all regular $B$-quantizations, resp. $(B / h B)$-quantization of the restricted Poisson scheme $X_{o}$. Equivalently, $Q\left((B / h B), X_{o}\right)$ is the set of isomorphism classes of restricted Poisson deformations $X / S$ of $X_{o}$. Reduction modulo $h$ defines a map

$$
\sigma: Q\left(B, X_{o}\right) \rightarrow Q\left((B / h B), X_{o}\right) .
$$

The given family $X / S$ defines a point $p \in Q\left((B / h B), X_{o}\right)$, and we have to prove that the preimage $\sigma^{-1}(p) \subset Q\left(B, X_{o}\right)$ is not empty and is a torsor over $H^{1}\left(X, \mathcal{H}_{\text {log }}\right)$. Indeed, denote $I=\mathfrak{m}_{B} / \mathfrak{m}_{B}^{2}$ and $I_{0}=\mathfrak{m}_{B / h B} / \mathfrak{m}_{B / h B}^{2}$. We have a short exact sequence

$$
0 \longrightarrow h \cdot k \longrightarrow I \longrightarrow I_{0} \longrightarrow 0
$$

of small Dieudonné modules, and Definition 1.9 implies in particular that $K(h)=$ $h^{[p]}=h$, so that the module on the left-hand side is $k\langle 1\rangle$. Therefore we have a short exact sequence of sheaves

$$
0 \longrightarrow \mathcal{H}_{\text {log }} \longrightarrow \mathcal{H}\langle I\rangle \longrightarrow \mathcal{H}\left\langle I_{0}\right\rangle \longrightarrow 0
$$

on $X$, and by Proposition $1.22, Q\left(B, X_{o}\right)$ and $X\left((B / h B), X_{o}\right)$ are naturally isomorphic to the first étale cohomology group of $X$ with coefficients in the second and the third term of (1.13). Thus to prove the claim, it suffices to show that taking first cohomology preserves the exactness of (1.13). For this it suffices to prove that (1.13) splits, and it further suffices to prove that (1.12) splits. This is well-known: $k\langle 1\rangle$ is an injective object in the category of small Dieudonné modules over $k$ (see, e.g., $[\mathrm{DP}])$.

Theorem 1.23 is analogous to [BK1, Theorem 1.8] in the characteristic 0 case. We note that in characteristic 0 , one has to consider information on all levels to obtain a full parameterization of quantizations. In positive characteristic, we cannot really do it; we restrict our attention to Frobenius-constant quantizations, impose a stronger admissibility condition, and extension to higher levels becomes automatic and unique. Unfortunately, if a symplectic manifold $X$ satisfying assumptions of Theorem 1.23 is projective over $k$, then we must have $[\Omega]=0$, which is impossible. Therefore, as we have already noted in Remark 1.14, our methods do not apply well enough to projective symplectic manifolds such as surfaces of type $K 3$. Most probably, such manifolds do not admit Frobenius-constant quantizations at all.

Our final result concerns the particular case of Theorem 1.23 when $B=k[[h]]$, $S=$ Spec $k$, so that $X=X_{o}$ is itself admissible (in particular, it is reduced, and we have $\left.X^{[p]}=X^{(1)}\right)$. In this case, the torsor in Theorem 1.23 is obviously split, so that we have a natural isomorphism

$$
Q(k[[h]], X) \cong H_{\text {et }}^{1}\left(X, \mathcal{H}_{\text {log }}\right) .
$$

In other words, every regular Frobenius-constant quantization $\mathcal{O}_{h}$ of $X$ is uniquely characterized by a cohomology class $\left[\mathcal{O}_{h}\right] \in H^{1}\left(X, \mathcal{H}_{\text {log }}\right)$. The cohomology long 
exact sequence associated to (1.11) allows one to give a more precise description of the group $H_{\text {et }}^{1}\left(X, \mathcal{H}_{\text {log }}\right)$ : we have a short exact sequence

$$
0 \longrightarrow \operatorname{Pic}(X) / p \operatorname{Pic}(X) \longrightarrow H_{e t}^{1}\left(X, \mathcal{H}_{l o g}\right) \stackrel{\mathrm{Br}}{\longrightarrow} \operatorname{Br}_{p}(X) \longrightarrow 0,
$$

where $\operatorname{Pic}(X)=H_{e t}^{1}\left(X, \mathcal{O}_{X}^{*}\right)$ is the Picard group of $X$, and $\operatorname{Br}_{p}(X) \subset H_{e t}^{2}\left(X, \mathcal{O}_{X}^{*}\right)$ is the $p$-torsion part of the Brauer group. By projection, the characterizing class $\left[\mathcal{O}_{h}\right] \in H_{e t}^{1}\left(X, \mathcal{H}_{\text {log }}\right)$ of every regular Frobenius-constant quantization $\mathcal{O}_{h}$ of $X$ gives a $p$-torsion element $\operatorname{Br}\left(\left[\mathcal{O}_{h}\right]\right)$ in the Brauer group.

This has the following interpretation in terms of quantizations. Consider the scheme $\widehat{X}=\operatorname{Spec} \mathcal{O}_{X}^{p}[[h]] ;$ this is essentially the completion of $X^{(1)} \times \operatorname{Spec} k[[h]]$ along the special fiber $X^{(1)} \subset X^{(1)} \times$ Spec $k[[h]]$, but it exists as an actual scheme over Spec $k[[h]]$ rather than simply a formal scheme, so that the complement $\bar{X}=$ $\widehat{X} \backslash X^{(1)}$ is a well-defined scheme as well. Denote by $\rho: \bar{X} \rightarrow X^{(1)}$ the projection. By [EGA, III, Théorème 5.4.5], the category of coherent sheaves on $\widehat{X}$ is equivalent to the category of complete finitely generated topological sheaves of $\mathcal{O}_{X}^{p}[[h]]$-modules on $X^{(1)}$. In particular, any Frobenius-constant quantization $\mathcal{O}_{h}$ of $X$ defines a coherent sheaf of algebras on $\widehat{X}$.

Proposition 1.24. For any central quantization $\mathcal{O}_{h}$ of a symplectic manifold $X / S$, the algebra $\mathcal{O}_{h}\left(h^{-1}\right)$ is a simple algebra over the localization $\mathcal{Z}_{h}\left(h^{-1}\right)$ of its center $\mathcal{Z}_{h}$. Moreover, assume that $S=$ Spec $k$ and that we have $H^{2}\left(X, \mathcal{O}_{X}\right)=0$. Then for any regular Frobenius constant quantization $\mathcal{O}_{h}$ of $X$, the class of the Azumaya algebra $\mathcal{O}_{h}\left(h^{-1}\right)$ in the Brauer group $\operatorname{Br}(\bar{X})$ is equal to the pullback $\rho^{*} \operatorname{Br}\left(\left[\mathcal{O}_{h}\right]\right)$ of the projection $\operatorname{Br}\left(\left[\mathcal{O}_{h}\right]\right)$ of the characterising class $\left[\mathcal{O}_{h}\right] \in H_{\text {et }}^{1}\left(X, \mathcal{H}_{\text {log }}\right)$.

This is proved in Subsection 4.2 on page 436. In particular, this means that an admissible $X / k$ has quantizations which give a split Azumaya algebra on $\bar{X}$.

1.5. Conjectures. We would like to finish the section with some speculations motivated by Lemma 1.3. Namely, we propose the following.

Conjecture 1.25. Let $B$ be a local commutative algebra over $k$ with maximal ideal $\mathfrak{m} \subset B$, and let $\widetilde{A}$ be a flat associative algebra over $B$ such that $A=\widetilde{A} / \mathfrak{m}$ is commutative. Then there exist a canonical subalgebra $\widetilde{A}^{p} \subset \widetilde{A}$ such that $\widetilde{A}^{p}$ is flat over $B^{p} \subset B$, the natural map $\widetilde{A}^{p} \otimes_{B^{p}} B \rightarrow \widetilde{A}$ is injective, and the quotient map $\widetilde{A} \rightarrow A$ induces an isomorphism between $\widetilde{A}^{p} / \mathfrak{m}^{p} \cong\left(\widetilde{A}^{p} \otimes_{B^{p}} B\right) / \mathfrak{m}$ and $A^{p} \subset A$.

This conjecture does hold in the two extreme cases of commutative deformations $\widetilde{A}\left(\widetilde{A}^{p}\right.$ is the subalgebra generated by $p$-th powers) and of central quantizations of symplectic affine manifolds $\left(\widetilde{A}^{p}\right.$ is the center of the algebra $\left.\widetilde{A}\right)$. Lemma 1.3 is Conjecture 1.25 for $B=k[h] / h^{p-1}$ (in this case $B^{p}=k$ ). The conjecture essentially says that even in the general case, $A^{p} \subset A$ does extend to a flat subalgebra in the whole $\widetilde{A}$, possibly no longer central nor commutative, and this flat deformation of $A^{p}$ is moreover induced from $B^{p} \subset B$.

If one thinks in terms of analogy between deformations and automorphisms, then Conjecture 1.25 is analogous to the fact that the automorphism group Aut $A$ preserves $A^{p} \subset A$, and its induced action on $A^{p}$ factors through the Frobenius map of the natural group scheme structure. Of course, this analogy is flimsy at best, so that we have no really convincing reason to believe that the conjecture is true. If it is true, its proof might possibly involve an extension of the restricted Lie 
algebra structure on vector fields to some sort of structure on the whole Hochschild cohomology DG Lie algebra of a commutative algebra $A$.

If one assumes Conjecture 1.25, then the natural generalization of Theorem 1.23 would replace Frobenius-constant quantizations with all quantizations $\widetilde{A}$ with prescribed algebra $\widetilde{A}^{p}$. Alternatively, one might want to study quantizations such that $\widetilde{A}^{p} \cong \widetilde{A}$. The latter would correspond to the condition $C(\Omega)=\Omega$ in Theorem 1.11, and as we noted in Remark 1.14, it seems a more natural question for compact manifolds, such as even-dimensional abelian varieties, or surfaces of type $K 3$.

\section{Poisson structures}

2.1. Central quantizations. The goal of this section is to prove Theorem 1.11 and Theorem 1.12. We start with some preliminaries on differential geometry in positive characteristic.

Let $B$ be a Noetherian commutative algebra over the field $k$, and let $A$ be a flat quasiregular commutative algebra over $B$. Denote by $A^{[p]}=A^{p} \otimes_{B^{p}} B \subset A$ the $B$-subalgebra generated by $p$-th powers of elements in $A$; the algebra $A^{[p]}$ is a quotient of the pullback $A \otimes \mathrm{Fr}_{B} B$ of the algebra $A$ with respect to the Frobenius map $\operatorname{Fr}_{B}: B \rightarrow B$, and the Frobenius map $\operatorname{Fr}_{A}: A \rightarrow A$ factors through a natural map map $\operatorname{Fr}_{A}[p]: A \rightarrow A^{[p]}$. Denote by $\Omega^{*}(A)=\Lambda^{\bullet} \Omega^{1}(A / B)$ the modules of differential forms on $A / B$, and denote by $\Omega_{c l}^{\bullet}(A) \subset \Omega^{\bullet}(A)$ the subspaces of closed forms. Denote $\Omega_{[p]}^{\bullet}(A)=\Omega^{\bullet}(A / B) \otimes_{\mathrm{Fr}_{A}[p]} A^{[p]}$, and denote by $C^{\bullet}: \Omega_{c l}^{\bullet}(A) \rightarrow \Omega_{[p]}^{\bullet}(A)$ the $A^{[p]}$-linear Cartier operations.

Consider the module $T(A)=T(A / B)$ of $B$-linear derivations of the algebra $A$ (in geometric language, these are relative vector fields on $\operatorname{Spec} A$ over $\operatorname{Spec} B$ ). It is a flat $A$-module and a Lie algebra; moreover, for any derivation $\xi: A \rightarrow A$, its $p$-th power $\xi^{p}: A \rightarrow A$ is also a derivation, so that $T(A)$ is a restricted Lie algebra. The Lie algebra $T(A)$ acts on $\Omega^{\bullet}(A)$ by the Lie derivative, and we will denote the action of a vector field $\xi \in T(A)$ by ad $\xi: \Omega^{\bullet}(A) \rightarrow \Omega^{\bullet}(A)$.

We note that by extension of scalars every differential form $\alpha \in \Omega^{l}(A)$ on $A / B$ induces a form $\operatorname{Fr}_{A[p]}^{*} \alpha \in \Omega_{[p]}^{l}(A)$. In particular, a function $f \in A$ is a differential form of degree 0 , and we have $\operatorname{Fr}_{A^{[p]}}^{*} f=f^{p} \in A^{p}$. Again by extension of scalars, every vector field $\xi \in T(A)$ induces a contraction operation $\alpha \mapsto \alpha\lrcorner \xi, \Omega_{[p]}^{\bullet+1}(A) \rightarrow$ $\Omega_{[p]}^{\bullet}(A)$.

We will need one fact on the relation between Cartier operations and the restricted Lie algebra structure on $T(A)$. Namely, for any vector field $\xi \in T(A)$ define an operation $i_{\xi}^{[p]}: \Omega^{\bullet+1}(A) \rightarrow \Omega^{\bullet}(A)$ by

$$
\left.\left.i_{\xi}^{[p]}(\alpha)=\xi^{[p]}\right\lrcorner \alpha-(\operatorname{ad} \xi)^{p-1}(\xi\lrcorner \alpha\right) .
$$

By the Cartan homotopy formula, the operation $i_{\xi}^{[p]}$ commutes with the de Rham differential, so that it induces an operation on the de Rham cohomology groups $H_{D R}^{\bullet}(A) \cong \Omega^{\bullet}\left(A^{p}\right)$.

Lemma 2.1. For every closed form $\alpha \in \Omega_{c l}^{\bullet}(A)$ we have

$$
\left.C^{\bullet-1}\left(i_{\xi}^{[p]}(\alpha)\right)=C \cdot(\alpha)\right\lrcorner \xi .
$$


Proof. Both sides are additive and $A^{p}$-linear with respect to $\alpha$. Moreover, for any closed forms $\alpha, \beta$ we have

$$
\left.\left.(\xi\lrcorner \alpha) \wedge \operatorname{ad} \xi(\beta)=(-1)^{\operatorname{deg} \alpha} \operatorname{ad} \xi(\alpha) \wedge(\xi\lrcorner \beta\right)-d((\xi\lrcorner \alpha) \wedge(\xi\lrcorner \beta\right) ;
$$

therefore

$$
\begin{aligned}
i_{\xi}^{[p]}(\alpha \wedge \beta)- & i_{\xi}^{p} \alpha \wedge \beta+\alpha \wedge i_{\xi}^{p} \beta \\
= & \left.\sum_{i=1}^{p-2}\left(\begin{array}{c}
p-1 \\
i
\end{array}\right)(\xi\lrcorner(\operatorname{ad} \xi)^{i} \alpha\right) \wedge(\operatorname{ad} \xi)^{p-1-i} \beta \\
& \left.\quad+(-1)^{\operatorname{deg} \alpha} \sum_{i=1}^{p-2}\left(\begin{array}{c}
p-1 \\
i
\end{array}\right)(\operatorname{ad} \xi)^{i} \alpha \wedge(\xi\lrcorner(\operatorname{ad} \xi)^{p-1-i} \beta\right) \\
= & \left.\sum_{i=1}^{p-2}\left(\left(\begin{array}{c}
p-1 \\
i
\end{array}\right)+\left(\begin{array}{c}
p-1 \\
i+1
\end{array}\right)\right)(\xi\lrcorner(\operatorname{ad} \xi)^{i} \alpha\right) \wedge(\operatorname{ad} \xi)^{p-1-i} \beta \\
& \left.\left.\quad+d\left((\operatorname{ad} \xi)^{p-2}((\xi\lrcorner \alpha) \wedge(\xi\lrcorner \beta\right)\right)\right) \\
= & \left.\left.d\left((\operatorname{ad} \xi)^{p-2}((\xi\lrcorner \alpha) \wedge(\xi\lrcorner \beta\right)\right)\right),
\end{aligned}
$$

which implies that the map $H_{D R}^{\bullet+1}(A) \rightarrow H_{D R}^{\bullet}(A)$ induced by $i_{\xi}^{[p]}$ is a derivation with respect to the algebra structure on $H_{D R}^{\cdot}$. Thus it suffices to prove $(2.1)$ for forms $\alpha$ of degree 1 . Let $\alpha$ be such a form, consider the connection $\nabla=d+\alpha$ on the $A$-module $A$, and denote by $f: A \rightarrow A$ multiplication by $\alpha\lrcorner \xi$. By definition of the Cartier operator, $C^{1}(\alpha)-\mathrm{Fr}_{A[p]}^{*} \alpha$ is the $p$-curvature of the connection $\nabla$; in other words, we have

$$
\left.\left.\left.\left(\nabla_{\xi}\right)^{p}=\nabla_{\xi^{[p]}}+C^{1}(\alpha)\right\lrcorner \xi-\operatorname{Fr}_{A[p]}^{*}(\alpha\lrcorner \xi\right)=\nabla_{\xi^{[p]}}+C^{1}(\alpha)\right\lrcorner \xi-f^{p} .
$$

Since $\nabla_{\xi}=\xi+f,(2.1)$ is therefore equivalent to

$$
\left.(\xi+f)^{p}=\xi^{p}+\alpha\right\lrcorner \xi^{[p]}+f^{p}-i_{\xi}^{[p]}(\alpha) .
$$

This immediately follows from Lemma 1.6: take $x=\xi, y=f$, and consider the Lie algebra $L$ of differential operators $A \rightarrow A$ of order $\leq 1$ with the natural filtration by order (inverted and shifted by 1 ).

Assume now that the quasiregular algebra $A / B$ is equipped with a non-degenerate closed 2-form $\Omega \in \Omega_{c l}^{2}(A)$. As in characteristic 0 , the form $\Omega$ induces a $B$-linear Poisson bracket on the algebra $A$. This structure is non-degenerate in the sense that the Poisson bivector $\Theta \in \Lambda^{2}(T(A))$ is a non-degenerate bilinear form on the $A$-module $\Omega^{1}(A)$; conversely, every non-degenerate $B$-linear Poisson bracket on $A$ comes from a symplectic form. For any $f \in A$ we denote the derivation $a \mapsto\{a, f\}$ by $H_{f}$; explicitly, we have $\left.H_{f}\right\lrcorner \Omega=d a$. Vector fields $H_{f}$ are called Hamiltonian and form a Lie subalgebra $H(A) \subset T(A)$ in the Lie algebra $T(A)$.

Corollary 2.2. The subalgebra $H(A)$ is closed under the restricted Lie algebra structure if and only if $C^{2}(\Omega)=0$.

Proof. By definition, for any $f \in A$ we have $\left.C^{1}\left(H_{f}\right\lrcorner \Omega\right)=C^{1}(d f)=0$. Lemma 2.1 yields

$$
\left.\left.C^{1}\left(H_{f}^{[p]}\right\lrcorner \Omega\right)=H_{f}^{(1)}\right\lrcorner C^{2}(\Omega) .
$$


The vector field $H_{f}^{[p]}$ is Hamiltonian if and only if the left-hand side vanishes; therefore $H(A) \subset T(A)$ is closed under the restricted Lie algebra structure if and only if the right-hand side vanishes for any $f$. Since $\Omega$ is non-degenerate, Hamiltonian vector fields generate $T(A)$ as an $A$-module, so that this is possible if and only if $C^{2}(\Omega)=0$.

The proof of Theorem 1.11. All claims are local, therefore we can assume that $X=$ $\operatorname{Spec} A$ and $S=\operatorname{Spec} B$ are affine. Then equivalence between (i) and (ii) becomes Corollary 2.2. To prove the second claim, we can assume that we are given a quasiregular $B$-algebra $A$ equipped with a non-degenerate closed 2-form $\Omega \in \Omega_{c l}^{2}(A)$, and its quantization $A_{h}$ such that the center $\mathcal{Z}_{h} \subset A_{h}$ satisfies $\mathcal{Z}_{h} / h \cong \mathcal{Z} \subset A$. Moreover, by Lemma 1.10 the Poisson center $\mathcal{Z} \subset A$ coincides with the subalgebra $A^{[p]} \subset A$. Consider the quotient $A_{h} / h^{p}$, take an element $f \in A$ and lift $f^{p} \in A^{[p]}$ to an element $\widetilde{f}^{p} \in \mathcal{Z}_{h}$. By Lemma 1.3 we have

$$
\widetilde{f}^{p}=f^{p}+f_{1} h+\cdots+f_{p-2} h^{p-2}+g h^{p-1} \bmod h^{p}
$$

for some $f_{1}, \ldots, f_{p-2} \in A^{[p]}$ and $g \in A$. By $(1.1),\left[f_{i}, y\right]$ is divisible by $h^{p-1}$ for all $f_{i}$ and any $y \in A$, and the same is true for $f^{p}$. On the other hand, since $\tilde{f}^{p}$ is central, we have $\left[\widetilde{f}^{p}, y\right]=0$; reducing this modulo $h^{p+1}$, we obtain

$$
(\operatorname{ad} f)^{p}(y)+[g, y] h^{p-1}=0 \bmod h^{p+1},
$$

which further reduces to $H_{f}^{[p]}(y)=H_{g}(y)$ and $H_{f}^{[p]}=H_{g}$. This finishes the proof.

2.2. Conformal derivations. Having proved Theorem 1.11, we now proceed to Theorem 1.12 .

Definition 2.3. Assume given a Poisson algebra $A / k$ and a constant $\lambda \in k$. A conformal derivation $\xi$ of the algebra $A$ with weight $\lambda$ is a $k$-linear map $\xi: A \rightarrow A$ which is a derivation with respect to the multiplication in $A$ and satisfies

$$
\xi(\{x, y\})=\{\xi(x), y\}+\{x, \xi(y)\}-\lambda\{x, y\} .
$$

A conformal derivation of weight 0 is just a Poisson derivation, in particular, all Hamiltonian derivations are conformal of weight 0 .

Lemma 2.4. Assume given a Poisson algebra $A / k$ and a conformal derivation $\xi: A \rightarrow A$ of some weight $\lambda \in k$. Then for any two elements $x, y \in A$ we have

$$
\begin{aligned}
\xi(L(x, y))= & \lambda L(x, y)+\operatorname{ad}(x+y)^{p-1}(\xi(x+y)) \\
& -\operatorname{ad}(x)^{p-1}(\xi(x))-\operatorname{ad}(y)^{p-1}(\xi(y)), \\
\xi(P(x, y))= & \lambda P(x, y)+\operatorname{ad}(x y)^{p-1}(\xi(x y)) \\
& -x^{p} \operatorname{ad}(y)^{p-1}(\xi(y))-y^{p} \operatorname{ad}(x)^{p-1}(\xi(x)),
\end{aligned}
$$

where we denote $\operatorname{ad}(z)(w)=\{z, w\}$.

Proof. We pass to the universal situation. Consider the algebra $Q^{\bullet}(x, y, z)$ of quantized polynomials in three variables $x, y, z$. By definition, the localization algebra $Q^{\bullet}(x, y, z)\left(h^{-1}\right)$ is just the free associative $k\left[h, h^{-1}\right]$-algebra generated by $x, y$ and $z$; therefore there exists a unique derivation $e: Q^{\bullet}(x, y, z)\left(h^{-1}\right) \rightarrow Q^{\bullet}(x, y, z)\left(h^{-1}\right)$ which annihilates $x, y$ and $z$ and satisfies $e(h)=\lambda h$. This derivation obviously preserves the subalgebra $Q^{\bullet}(x, y, z) \subset Q^{\bullet}(x, y, z)\left(h^{-1}\right)$. Consider the derivation 
$\xi=e+\operatorname{ad}(z)$ of the algebra $Q^{\bullet}(x, y, z)$. Define a grading on $Q^{\bullet}(x, y, z)$ by setting $\operatorname{deg} x=\operatorname{deg} y=\operatorname{deg} h=0, \operatorname{deg} z=1$, and let $\widetilde{Q}^{\bullet}(x, y)$ be the component of degree 0 . The derivation $\xi$ and the quantized algebra structure are compatible with the grading and restrict to $\widetilde{Q}^{\bullet}(x, y) \subset Q^{\bullet}(x, y, z)$.

The quotient $\bar{Q}^{\bullet}(x, y)=\widetilde{Q}^{\bullet}(x, y) / h$ of the algebra $\widetilde{Q}^{\bullet}(x, y)$ is a Poisson algebra equipped with a derivation $\xi$, and since $e(h)=\lambda h$, the derivation $\xi$ is conformal of weight $\lambda$. It is easy to check that the Poisson algebra $\bar{Q}^{\circ}(x, y)$ is freely generated by $\xi^{l}(x)$ and $\xi^{l}(y), l \geq 0$. Therefore for any two elements $x, y \in A$ in a Poisson algebra $A$ equipped with a conformal derivation $\xi$ of weight $\lambda$, there exists a unique Poisson map $s: \bar{Q}^{\bullet}(x, y) \rightarrow A$ which sends $x$ to $x, y$ to $y$, and satisfies $w(\xi(a))=\xi(w(a))$ for any $a \in \bar{Q}^{\circ}(x, y)$.

We conclude that it suffices to prove the claim for $A=\bar{Q}^{\bullet}(x, y)$. Moreover, it even suffices to prove it for the quantized subalgebra $\widetilde{Q}^{\bullet}(x, y) \subset Q^{\bullet}(x, y, z)$. But since the algebra $Q^{\bullet}(x, y, z)$ has no $h$-torsion, we may multiply both sides of $(2.3)$ by $h^{p-1}$; both equations then immediately follow from (1.1) and (1.3).

2.3. Restricted Poisson structures. Return to the notation of Subsection 2.1: $A$ is a quasiregular algebra over $B$ with a non-degenerate $B$-linear Poisson structure induced by a symplectic form $\Omega \in \Omega^{2}(A)$. By Lemma 1.10, the subalgebra $A^{[p]} \subset A$ is the Poisson center of the algebra $A$.

Lemma 2.5. Assume given a restricted structure on the Poisson algebra A. Then a Poisson derivation $\xi \in T(A)$ is Hamiltonian if and only if we have

$$
\xi\left(a^{[p]}\right)=\operatorname{ad}(a)^{p-1}(\xi(a))
$$

for any $a \in A$.

Proof. Since $\xi$ is Poisson, the 1 -form $\beta=\xi\lrcorner \Omega$ is closed, $C^{1}(\beta)$ is well-defined, and $\xi$ is Hamiltonian if and only if $C^{1}(\beta)=0$. Since Hamiltonian vector fields generate the $A$-module $T(A)=T(A / B)$, this holds if and only if

$$
\left.H_{a}^{(1)}\right\lrcorner C^{1}(\beta)=0
$$

for any Hamiltonian vector field $H_{a}, a \in A$. By Lemma 2.1, this is equivalent to

$$
C^{0}\left(i_{H_{a}}^{[p]}(\beta)\right)=0
$$

and since the Cartier operation $C^{0}$ is injective, this reduces to $i_{H_{a}}^{[p]}(\beta)=0$, which by definition means exactly (2.4).

Proposition 2.6. Assume given a conformal derivation $\xi: A \rightarrow A$ of weight $\lambda$ of a Poisson algebra $A$.

(i) For any restricted structure on $A$, the map $\kappa: A \rightarrow A$ given by

$$
\kappa(a)=(\xi-\lambda \mathrm{id})\left(a^{[p]}\right)-\operatorname{ad}(a)^{p-1}(\xi(a))
$$

is a Frobenius derivation of the algebra $A$ with values in the Poisson center $A^{[p]} \subset A$.

(ii) If $\lambda=1$, then every Frobenius derivation $\kappa: A \rightarrow A^{[p]} \subset A$ is given by (2.5) for a unique restricted structure on the Poisson algebra $A$. 
Proof. To prove (i), we apply (2.3) and deduce that $\kappa(a+b)=\kappa(a)+\kappa(b)$ and $\kappa(a b)=a^{p} \kappa(b)+b^{p} \kappa(a)$. Moreover, for any $a, b \in A$ we have

$$
\begin{aligned}
\{\kappa(a), b\} & =\xi\left(\left\{a^{[p]}, b\right\}\right)-\left\{a^{[p]}, \xi(b)\right\}-\left[\xi, H_{a}^{[p]}\right](b) \\
& =\xi\left(H_{a}^{[p]}(b)\right)-H_{a}^{[p]}(\xi(b))-\left[\xi, H_{a}^{[p]}\right](b)=0,
\end{aligned}
$$

so that $\kappa(a)$ indeed lies in the Poisson center $A^{[p]} \subset A$.

To prove (ii), denote $\alpha=\xi\lrcorner \Omega$. By the Cartan homotopy formula, $d \alpha=\lambda \Omega$, and (2.5) can be rewritten as

$$
a^{[p]}=i_{H_{a}}^{[p]} \alpha-\kappa(a) .
$$

Therefore the map $a \mapsto a^{[p]}$ is indeed uniquely defined by $\xi$ and $\kappa$, and we have to prove that it satisfies (1.4), (1.5), and (1.6). Equation (1.4) is immediate: since $d \kappa(a)=0$, we have $\left.d a^{[p]}=d i_{H_{a}}^{[p]} \alpha=H_{a}^{[p]}\right\lrcorner \Omega$. To prove the rest, note that any function $f \in A$ which commutes with all Hamiltonian derivations lies in the Poisson center $A^{[p]} \subset A$. For such a function, we have $\xi(f)=0$ and $(\xi-$ id $)(f)=-f$. Therefore it suffices to prove both (1.5) and (1.6) after applying to both sides the map $(\xi-\mathrm{id})$ and the maps $H_{a}$ for all $a \in A$. Both equations then immediately follow from (2.5) and (2.3).

Proof of Theorem 1.12. The claim is local in Zariski topology, so that we can assume that $S=\operatorname{Spec} B$ and $X=\operatorname{Spec} A$ are affine, with $A$ quasiregular over $B$. We are given a Frobenius derivation $K: B \rightarrow B$, and we have to establish a bijection between the set of all restricted structures on the Poisson algebra $A$ which are compatible with $K: B \rightarrow B$ and the set of all isomorphisms between $\Omega_{[p]}^{1}(A)$ torsors $C_{*}^{1}\left[\mathcal{M}_{\Omega}\right]$ and $\left.\operatorname{Fr}_{A[p]}^{*} \Omega\right\lrcorner\left[\theta_{K}\right]$. By the Cartan homotopy formula, sections of the torsor $\left[\mathcal{M}_{\Omega}\right]$ correspond to conformal derivations $\xi: A \rightarrow A$ of weight 1 . By Proposition 2.6 (i), every restricted structure on $A$ induces a map from the set of all conformal derivations $\xi: A \rightarrow A$ of weight 1 to the set of all Frobenius derivations $\kappa: A \rightarrow A^{[p]} \subset A$. It immediately follows from (2.5) that no matter what $\xi$ one takes, a restricted structure is compatible with $K: B \rightarrow B$ if and only if the corresponding $\kappa: A \rightarrow A^{[p]} \subset A$ is equal to $K$ on $B \subset A$.

But the $B$-modules of $B$-linear Frobenius derivations $A \rightarrow A^{[p]} \subset A$ are naturally identified with $\Omega_{[p]}^{1}(A)$ by $\left.\xi \mapsto \operatorname{Fr}_{A^{[p]}}^{*} \Omega\right\lrcorner \xi$. Therefore the torsor $\left.\operatorname{Fr}_{A^{[p]}}^{*} \Omega\right\lrcorner\left[\theta_{K}\right]$ is naturally identified with the set of all Frobenius derivations $\kappa: A \rightarrow A^{[p]} \subset A$ which are equal to $K$ on $B \subset A$.

In other words, a restricted structure is compatible with $K$ if and only if the correspondence $\xi \mapsto \kappa$ actually defines a map $\left.\left[\mathcal{M}_{\Omega}\right] \rightarrow \operatorname{Fr}_{A[p]}^{*} \Omega^{(1)}\right\lrcorner\left[\theta_{K}\right]$. By Lemma 2.5, two conformal derivations give the same $\kappa$ if and only if their difference is a Hamiltonian derivation; by the Cartan homotopy formula, this means that this map actually factors through the quotient $C_{*}^{1}\left[\mathcal{M}_{\Omega}\right]=\left[\mathcal{M}_{\Omega}\right] / d(A)$ and gives an isomorphism $\left.C_{*}^{1}\left[\mathcal{M}_{\Omega}\right] \cong \Omega^{(1)}\right\lrcorner\left[\theta_{K}\right]$. Finally, by Proposition 2.6 (ii), every such isomorphism comes from a unique restricted structure on $A$ compatible with $K: B \rightarrow B$.

Remark 2.7. Informally, the situation can be described as follows. There are three natural $\Omega_{[p]}^{1}(A)$-torsors associated to $A / B$ and $K: B \rightarrow B$. First, there is a torsor of all $B$-linear conformal derivations of weight 1 , considered modulo Hamiltonian derivations. This torsor does not depend on $K$. Second, there is the torsor $\left.\operatorname{Fr}_{A[p]}^{*} \Omega\right\lrcorner\left[\theta_{K}\right]$, which is identified with the set of all Frobenius derivations 
$A \rightarrow A^{[p]} \subset A$ which are equal to $K$ on $B \subset A$. This torsor, in spite of notation, does not depend on the symplectic form $\Omega$. Finally, there is the set of restricted structures on $A$ compatible with $K: B \rightarrow B$, and this set is also a torsor over $\Omega_{A[p]}^{1}$ - recall (page 414, remarks after Definition 1.9) that the difference of two restricted structures is a Frobenius derivation $A \rightarrow A$ with values in the Poisson center $A^{[p]} \subset A$. Proposition 2.6 shows that the sum of these three torsors is canonically trivialized. Therefore giving an element in one of them is equivalent to giving an isomorphism between the other two.

\section{LOCAL THEORY}

To proceed further, we need to study symplectic manifolds and their quantizations locally. In characteristic 0, "locally" means at best "in the formal neighborhood of a point". In positive characteristic, we can do better and replace a regular complete local algebra $A^{\prime}$ with its quotient $A=A^{\prime} / \mathfrak{m}^{[p]} \cdot A^{\prime}$ by the $p$-th powers of elements in the maximal ideal $\mathfrak{m} \subset A^{\prime}$. The algebra $A$ is a finite-dimensional local Artin algebra over $k$, and it is quasiregular. The goal of this section is to prove Proposition 1.18 and Proposition 1.19 - or rather, more precise versions of them which take account of the category structure - for $X=\operatorname{Spec} A$.

3.1. Differential geometry. Fix a finite-dimensional vector space $W$ over $k$. Consider the algebra $A=k\left[\left[W^{*}\right]\right] / \mathfrak{m}^{[p]}$, where $\mathfrak{m}^{[p]} \subset k\left[\left[W^{*}\right]\right]$ is the ideal generated by $a^{p}$ for all $a$ in the augmentation ideal $\mathfrak{m} \subset k\left[\left[W^{*}\right]\right]$. Then $A$ is a finite-dimensional local algebra over $k$, of rank $p^{\operatorname{dim} W}$, with maximal ideal $\mathfrak{m} \subset A$. Moreover, the algebra $A$ is quasiregular: the module $T(A) \cong W \otimes A$ of derivations of the algebra $A$ is a free $A$-module generated by the vector space $W$. Analogously, for every $k \geq 0$, the module $\Omega^{k}(A) \cong \Lambda^{k}\left(W^{*}\right) \otimes A$ of $k$-forms on $A$ is a free $A$-module generated by $\Lambda^{k}\left(W^{*}\right)$. The algebra $T(A)$ is a restricted Lie algebra; the Cartier operations $C^{k}$ induce graded ring isomorphisms $H_{D R}^{k}(A) \cong \Omega_{[p]}^{k}(A) \cong \Lambda^{k}\left(W^{*(1)}\right)$.

The group Aut $A$ of all automorphisms of the algebra $A$ has a natural structure of a quasiregular group scheme over $k$, with Lie algebra $T(A)$. The subgroup (Aut $A)_{0} \subset$ Aut $A$ of all automorphisms that preserve $\mathfrak{m} \subset A$ is a regular group scheme with Lie algebra $T(A)_{0}=W \otimes \mathfrak{m} \subset W \otimes A \cong T(A)$. The quotient homogeneous space (Aut $A) /(\text { Aut } A)_{0}$ is naturally identified with $\operatorname{Spec} A$.

Assume that $A$ is equipped with a non-degenerate closed 2-form $\Omega \in \Omega^{2}(A)$ (in particular, $W$ is even-dimensional). The form $\Omega$ defines a Poisson structure on the algebra $A$ and the subalgebra $H(A) \subset T(A)$ of Hamiltonian vector fields. We record the following obvious facts.

Lemma 3.1. The only Poisson ideals in $A$ are 0 and $A$ itself.

Proof. Since $A$ is local, any proper ideal $I \subset A$ lies in $\mathfrak{m}^{k}$ for some $k$; but since Hamiltonian vector fields generate $T(A)$ as an $A$-module, the ideal $I$ must be preserved by all derivations of the algebra $A$. An easy induction on $k$ shows that this is possible only for $I=0$.

Lemma 3.2. For any quantization $A_{h}$ of the algebra $A$, the algebra $A_{h}\left(h^{-1}\right)$ is an Azumaya algebra over the Laurent power series field $k((h))$.

Proof. By Lemma 1.10, the Poisson center of the algebra $A$ is $k \subset A$; therefore the algebra $A_{h}\left(h^{-1}\right)$ is central, and it remains to prove that it is a simple algebra. 
Indeed, let $I \subset A_{h}\left(h^{-1}\right)$ be a two-sided ideal. Then $I_{0}=I \cap A_{h}$ is a two-sided ideal in $A_{h} \subset A_{h}\left(h^{-1}\right)$, and $I=I_{0}\left(h^{-1}\right)$. We further note that by definition, $I_{0} / h \subset A$ is a Poisson ideal in $A$. By Lemma 3.1, this means that either $I_{0} / h=0$ or $I_{0} / h=A$. By the Nakayama Lemma, this implies that $I_{0}=0$ or $I_{0}=A_{h}$, which in turn yields $I=0$ or $I=A_{h}\left(h^{-1}\right)$.

Assume now that we are given a local Artin $k$-algebra $B$ equipped with a Frobenius derivation $K: B \rightarrow B$, and assume that the Poisson algebra $A \otimes B$ over $B$ is equipped with a restricted structure compatible with $K$. Denote by $\operatorname{Aut}(A)^{B}$ the algebraic group of all $B$-linear automorphisms of the algebra $A \otimes B$; it is obtained from $\operatorname{Aut}(A)$ by the Weil restriction of scalars from $B$ to $k$. The subgroup $\mathrm{G}^{B} \subset \operatorname{Aut}(A \otimes B / B)$ of automorphisms that preserve the (relative) symplectic form and the restricted structure inherits a structure of an algebraic group. We also have the subgroup $\operatorname{Aut}(A)_{0}^{B} \subset \operatorname{Aut}(A)^{B}$ of automorphisms that preserve the maximal ideal in $A \otimes B$; denote the intersection $\operatorname{Aut}(A)_{0}^{B} \cap \mathrm{G}^{B} \subset \operatorname{Aut}(A)^{B}$ by $\mathrm{G}_{B}^{0}$.

Lemma 3.3. $A$ B-linear derivation $\xi: A \otimes B \rightarrow A \otimes B$ lies in the Lie algebra of the subgroup $\mathrm{G}^{B}$ if and only if it is Hamiltonian, $\xi=H_{f}$ for some $f \in A \otimes B$.

Proof. To compute the Lie algebra of $\mathrm{G}^{B}$, consider the dual numbers algebra $k\langle\varepsilon\rangle=$ $k[\varepsilon] / \varepsilon^{2}$. By definition, a derivation $\xi$ comes from the Lie algebra of $\mathrm{G}^{B}$ if and only if $1+\xi \varepsilon: A \otimes B\langle\varepsilon\rangle \rightarrow A \otimes B\langle\varepsilon\rangle$ is compatible with the symplectic form and the restricted structure. Compatibility with the symplectic form means that $\xi$ is a Poisson derivation. Compatibility with the restricted structure reads as

$$
\begin{aligned}
a^{[p]}+\xi\left(a^{[p]}\right) \varepsilon & =(a+\xi(a) \varepsilon)^{[p]} \\
& =a^{[p]}+L(a, \xi(a) \varepsilon)+\xi(a)^{[p]} \varepsilon^{p}+\xi(a)^{p} \varepsilon^{[p]}+P(\xi(a), \varepsilon)
\end{aligned}
$$

for any $a \in A \otimes B$. By definition, $\varepsilon^{p}=\varepsilon^{[p]}=0$; moreover, since $\{-, \varepsilon\}$ vanishes tautologically, we have $P(\xi(a), \varepsilon)=0$. Therefore only the first two terms in the right-hand side are possibly non-trivial. Applying Lemma 1.6 to $A \otimes B \cdot \varepsilon \subset A \otimes B\langle\varepsilon\rangle$, we see that this is equivalent to (2.4), which by Lemma 2.5 holds if and only if $\xi$ is Hamiltonian.

Proposition 3.4. Assume given an Artin k-algebra $B$ and a Frobenius derivation $K: B \rightarrow B$. Then all the non-degenerate restricted Poisson structures on the algebra $A \otimes B$ over $B$ compatible with $K$ are isomorphic. Moreover, the group $\mathrm{G}_{0}^{B} \subset \operatorname{Aut}(A)^{B}$ is a regular algebraic group.

Proof. The set $R$ of all non-degenerate restricted Poisson structures on $A \otimes B$ over $B$ has a natural structure of an algebraic variety, and this variety is irreducible. Indeed, forgetting the restricted structure defines a map $R \rightarrow U \subset \Omega^{2}(A \otimes B)_{e x}$ onto the set $U$ of all non-degenerate $B$-linear Poisson structures on $A \otimes B$ which admit a restricted power operation, and by Theorem 1.11 the set $U$ is an open subset in the affine space of exact relative 2 -forms on $A \otimes B$ over $B$. On the other hand, by Theorem $1.12 R$ is an affine torsor over $U$ (with respect to $\Omega_{[p]}^{1}(A \otimes B$ ) considered as an additive algebraic group). Now, the group $\operatorname{Aut}(A)_{0}^{B}$ acts on $R$, and we have to show that this action is transitive. Since $U$ is irreducible, it suffices to show that the orbit $\operatorname{Aut}(A)_{0}^{B} \cdot r$ of any point $r \in R$ is the whole $R$. But by definition the stabilizer of a point $r \in R$ is $\mathrm{G}_{0}^{B}$, the automorphism group of $A \otimes B$ 
with the corresponding restricted Poisson structure. Therefore

$$
\begin{aligned}
\operatorname{dim} R & \geq \operatorname{dim} \operatorname{Aut}(A)_{0}^{B} \cdot r=\operatorname{dim} \operatorname{Aut}(A)_{0}^{B}-\operatorname{dim} \mathrm{G}_{0}^{B} \\
& \geq \operatorname{dim} \operatorname{Aut}(A)_{0}^{B}-\operatorname{dim} H_{0}(A \otimes B),
\end{aligned}
$$

where $H_{0}(A \otimes B)$ is the Lie algebra of the group $\mathrm{G}_{0}^{B}$ - which by Lemma 3.3 coincides with the Lie algebra of Hamiltonian vector fields on $A \otimes B$ preserving the maximal ideal. But

$$
\begin{aligned}
\operatorname{dim} R & =\operatorname{dim} U+\operatorname{dim} \Omega_{[p]}^{1}(A \otimes B) \\
& =\operatorname{dim} \Omega_{e x}^{2}(A \otimes B)+\operatorname{dim} H^{1}\left(\Omega^{\bullet}(A \otimes B)\right) \\
& =\operatorname{dim} \Omega^{1}(A \otimes B)-\operatorname{dim} \Omega_{e x}^{1}(A \otimes B),
\end{aligned}
$$

while on the other hand,

$$
\begin{aligned}
\operatorname{dim} \operatorname{Aut}(A)_{0}^{B}-\operatorname{dim} H_{0}(A \otimes B) & =\operatorname{dim} T(A \otimes B)-\operatorname{dim} H(A \otimes B) \\
& =\operatorname{dim} \Omega^{1}(A \otimes B)-\operatorname{dim} \Omega_{\text {ex }}^{1}(A \otimes B) .
\end{aligned}
$$

Therefore all the inequalities in (3.1) are in fact equalities. This means, first, that $\operatorname{dim} R=\operatorname{dim} \operatorname{Aut}(A)_{0}^{B} \cdot r$, so both sets coincide, and second, that $\operatorname{dim} \mathrm{G}_{0}^{B}$ is the same as the dimension of its Lie algebra, which means that the group $\mathrm{G}_{B}^{0}$ is regular.

Remark 3.5. If $B=k$, this proposition in particular implies that all symplectic forms $\Omega \in \Omega^{2}(A)$ with $C^{2}(\Omega)=0$ are isomorphic (every such form admits a compatible restricted power operation by Theorem 1.12). Since $C^{2}(\Omega)$ is an obvious invariant of a symplectic form, this is the best replacement for the Darboux Theorem one could hope for in characteristic $>0$.

3.2. Quantizations. We can now prove Proposition 1.18 for $X=\operatorname{Spec} A$. Assume given a quantization base $B_{0}$, a small extension $I \subset B \rightarrow B_{0}$, and a $B_{0}$-quantization $A_{0}$ of the algebra $A$. Denote by $\mathfrak{m}_{B} \subset B$ the maximal ideal of the local $k$-algebra $B$. Consider the category $Q\left(A_{0}, B\right)$ of all regular $B$-quantizations $A_{B}$ of the algebra $A$ equipped with an isomorphism $A_{B} / I A_{B} \cong A_{0}$. (We remind the reader that a $B$-quantization $A_{B}$ is called regular if and only if any $a \in A_{B}$ such that $a^{p}=0$ $\bmod \mathfrak{m}_{B} A_{B}$ satisfies $a^{p}=h^{p-1} a^{[p]}$.)

Proposition 3.6. All objects in the category $Q\left(A_{0}, B\right)$ are isomorphic.

Proof. Assume first that $B_{0}=k$, so that $I \subset B$ coincides with the maximal ideal $\mathfrak{m}_{B} \subset B$ and satisfies $\mathfrak{m}_{B}^{2}=0$. Assume also that $h=0$, so that a quantization $A_{B} \subset Q\left(A_{0}, B\right)$ is a restricted Poisson algebra and a square-zero extension of the restricted Poisson algebra $A_{0}=A$. We have to show that $A_{B} \cong A \otimes_{k} B$. Indeed, the algebra $A$ is by definition generated by $k=\operatorname{dim} W$ elements $a_{1}, \ldots, a_{k} \in A$ modulo the relations $a_{i}^{p}=0$. Lift the elements $a_{i}$ to elements $\widetilde{a}_{i} \in A_{B}$; since the quantization $A_{B}$ is regular, we still have $\widetilde{a}_{i}^{p}=h^{p-1} \widetilde{a}_{i}^{[p]}=0$. This means that $A_{B} \cong A \otimes B$ as commutative $B$-algebras. But by Proposition 3.4 all non-degenerate restricted Poisson algebra structures on $A \otimes B$ are isomorphic. It remains to notice that since $A_{B} / \mathfrak{m}_{B}$ is isomorphic to the non-degenerate Poisson algebra $A$, the Poisson structure on $A_{B}$ is also non-degenerate. 
Now consider the general case. For any two algebras $C_{1}, C_{2}$ equipped with surjective maps $C_{1} \rightarrow C, C_{2} \rightarrow C$ to a third algebra $C$, denote by $C_{1} \oplus^{C} C_{2}$ their Baer sum - that is, the subalgebra in $C_{1} \oplus C_{2}$ of elements with the same image in $C$. Assume given two different regular $B$-quantizations $A_{1}, A_{2} \in Q\left(A_{0}, B\right)$. Let $B^{\prime}=B \oplus^{B_{0}} B$, and let $A_{12}=A_{1} \oplus^{A_{0}} A_{2}$. The algebra $A_{12}$ is obviously a regular $B^{\prime}-$ quantization of the algebra $A$. Let $p_{1}: B^{\prime}=B \oplus^{B_{0}} B \rightarrow B$ be the projection onto the first summand, let $\delta: B \rightarrow B^{\prime}$ be the diagonal embedding, and let $\tau: B^{\prime} \rightarrow B^{\prime \prime}$ be the natural projection onto the quotient $B^{\prime \prime}=B^{\prime} / \delta\left(\mathfrak{m}_{B}\right)$. Then we obviously have $B^{\prime \prime} \cong k \oplus I$, and the map $p_{1} \oplus \tau: B^{\prime} \rightarrow B \oplus B^{\prime \prime}$ identifies the algebra $B^{\prime}$ with the Baer sum $B \oplus^{k} B^{\prime \prime}$. Under this identification, $A_{12} /(0 \oplus I) A_{12}$ is isomorphic to $A_{1}, A_{12}^{\prime}=A_{12} /\left(\mathfrak{m}_{B} \oplus 0\right) A_{12}$ is a regular $B^{\prime \prime}$-quantization of the algebra $A$, and we have $A_{12}=A_{1} \oplus^{A_{0}} A_{12}^{\prime}$.

Now, we have $I^{2}=0$ and $h=0$ in the algebra $B^{\prime \prime}=B^{\prime} / \delta\left(\mathfrak{m}_{B}\right)$; therefore, as we have already proved, $A_{12}^{\prime}$ must be isomorphic to $A \otimes B^{\prime \prime} \cong A \oplus I A$ as a restricted Poisson algebra. Therefore $A_{12} \cong A_{1} \oplus I A$ as restricted Poisson algebras. In particular, we have a restricted Poisson map $A_{1} \rightarrow A_{12}$; composing it with the natural projection $A_{12} \rightarrow A_{2}$ gives the required isomorphism $A_{1} \cong A_{2}$.

Corollary 3.7. Assume given a quantization $A_{1} \in Q\left(A_{0}, B\right)$, and consider the algebraic group Aut $\left(A_{1}\right)$ of all $B$-linear automorphisms of the restricted quantized algebra $A_{1}$. Then the natural reduction map Aut $\left(A_{1}\right) \rightarrow \operatorname{Aut}\left(A_{0}\right)$ is a surjective map of algebraic groups over $k$.

Proof. It suffices to prove that the map Aut $\left(A_{1}\right)\left(B^{\prime}\right) \rightarrow \operatorname{Aut}\left(A_{0}\right)\left(B^{\prime}\right)$ of groups of $B^{\prime}$-points is surjective for any $k$-algebra $B^{\prime}$. It is well-known that it suffices to prove it when $B^{\prime}$ is an arbitrary local Artin algebra, with maximal ideal $\mathfrak{m}_{B^{\prime}} \subset B^{\prime}$. By definition we have Aut $\left(A_{1}\right)\left(B^{\prime}\right) \cong \operatorname{Aut}\left(A_{1} \otimes B^{\prime}\right)$ and Aut $\left(A_{0}\right)\left(B^{\prime}\right) \cong \operatorname{Aut}\left(A_{0} \otimes B^{\prime}\right)$. Filtering $I \otimes B^{\prime} \subset B \otimes B^{\prime}$ by ideals $I \otimes \mathfrak{m}_{B^{\prime}}^{k} \subset I \otimes B^{\prime}$, we decompose the extension $B \otimes B^{\prime} \rightarrow B_{0} \otimes B^{\prime}$ into a series of small extensions. By induction, it therefore suffices to prove that for any small extension, the reduction map is surjective on the groups of points. In other words, we have to prove that any $g \in \operatorname{Aut}\left(A_{0}\right)$ can be lifted to an element $\widetilde{g} \in \operatorname{Aut}\left(A_{1}\right)$.

Indeed, let $A_{2} \in Q\left(A_{0}, B\right)$ be the algebra $A_{1}$ equipped with a different map $A_{1} \rightarrow A_{0}$ - namely, the composition of the original one with the automorphism $g: A_{0} \rightarrow A_{0}$. By Proposition 3.6, $A_{1}$ and $A_{2}$ are isomorphic in $Q\left(A_{0}, B\right)$; the isomorphism is the desired lifting $\widetilde{g} \in \operatorname{Aut}\left(A_{1}\right)$.

As an application of Proposition 3.6, we can extend Proposition 3.4 to the following uniqueness result.

Proposition 3.8. Assume given a quantization base B. There exists a unique, up to an isomorphism, regular $B$-quantization $A_{B}$ of the Poisson algebra $A$.

Proof. Uniqueness follows immediately by induction from Proposition 3.6. Moreover, it suffices to prove existence for $B=k[[h]]$ : for an arbitrary $B$, we can take $A_{B}=A_{k[[h]]} \otimes_{k[[h]]} B$. But by Proposition 3.4 we can assume that for some integer $n, A$ is the commutative algebra over $k$ generated by $x_{1}, \ldots, x_{n}, y_{n}, \ldots, y_{n}$ modulo the relations $x_{i}^{p}=y_{j}^{p}=0$, and the symplectic form $\Omega$ on $A$ is given by

$$
\Omega=d x_{1} \wedge d y_{1}+\cdots+d x_{n} \wedge d y_{n} .
$$


Then a regular $k[[h]]$-quantization of the algebra $A$ is given by the so-called reduced Weyl algebra $D$, which is generated by $x_{1}, \ldots, x_{n}, y_{1}, \ldots, y_{n}$ modulo the relations

$$
\begin{aligned}
x_{i} x_{j}-x_{j} x_{i} & =y_{i} y_{j}-y_{j} y_{i}=0, \\
x_{i} y_{j}-y_{j} x_{i} & =\delta_{i j} h, \\
x_{i}^{p}=y_{j}^{p} & =0,
\end{aligned}
$$

where $\delta_{i j}$ is the Kronecker delta symbol. It is immediately obvious that this quantization is regular and Frobenius-constant (the splitting map $s$ vanishes on all the generators $\left.x_{i}, y_{j}\right)$.

3.3. Automorphism groups. We finish this section with a more detailed description of the automorphism groups of quantizations. By Proposition 3.8, for any quantization base $B$ there exists a unique regular $B$-quantization of the algebra $A$; denote this $B$-quantization by $A_{B}$. Also denote by $\mathrm{G}^{B}=\operatorname{Aut}\left(A_{B}\right)$ the algebraic group of all $B$-linear automorphisms of the $B$-quantization $A_{B}$. If $B=k$, we have $\mathrm{G}^{k}=\mathrm{H}$, the group of Hamiltonian automorphisms of the restricted Poisson algebra A.

As in Subsection 1.4, for any small Dieudonné module $I$ over $k$ we denote by $\mathcal{H}(A, I)$ the kernel of the map $C^{1}-\mathrm{Fr}_{A[p]}^{*} \otimes K_{I}: \Omega_{c l}^{1}(A) \otimes I \rightarrow \Omega_{[p]}^{1}(A) \otimes I$, so that we have an exact sequence

$$
0 \longrightarrow \mathcal{H}(A, I) \longrightarrow \Omega_{c l}^{1}(A) \otimes I \stackrel{C^{1}-\mathrm{Fr}_{A[p]}^{*} \otimes K_{I}}{\longrightarrow} \Omega_{[p]}^{1}(A) \otimes I \longrightarrow 0
$$

of algebraic groups over $k$ (here we consider $k$-vector spaces $\Omega_{c l}^{1}(A) \otimes I$ and $\Omega_{[p]}^{1}(A)$ as additive algebraic groups, that is, we equip them with the scheme structure of an affine space, so that both become sums of several copies of $\mathbb{G}_{a}$ ). Note that by definition the group $\mathrm{H}$ acts naturally on the middle and the right-hand side term of this exact sequence, hence also on the group $\mathcal{H}(A, I)$.

Remark 3.9. As in Remark 1.17, we note that the map $\operatorname{Fr}_{A[p]}^{*} \otimes K_{I}$ is not $k$-linear, but only $k$-semilinear. Therefore the commutative algebraic group $\mathcal{H}(A, I)$ is not isomorphic to the sum of several copies of $\mathbb{G}_{a}$. In fact, even the group $\mathcal{H}(A, I)(k)$ of its $k$-valued points has no natural structure of a $k$-vector space. However, the algebraic group $\mathcal{H}(A, I)$ is a regular scheme over $k$.

Lemma 3.10. Assume given a quantization base $B_{0}$ and a small extension $I \subset$ $B \rightarrow B_{0}$. Then the kernel of the surjective map $\mathrm{G}^{B} \rightarrow \mathrm{G}^{B_{0}}$ is isomorphic to the algebraic group $\mathcal{H}(A, I)$, and the conjugation action of $\mathrm{G}^{B_{0}}$ on $\mathcal{H}(A, I)$ is induced from the natural action of $\mathrm{H}$ on $\mathcal{H}(A, I)$ via the reduction map $\mathrm{G}^{B_{0}} \rightarrow \mathrm{H}$.

Proof. As in the proof of Proposition 3.6, the general case immediately reduces to the case $B_{0}=k, \mathfrak{m}_{B}=I, \mathfrak{m}_{B}^{2}=0$ and $h=0$, so that $A_{B}$ is a restricted Poisson algebra over $B$. Simplify notation by setting $K=K_{I}$. For any $k$-algebra $B^{\prime}$, the group $\mathcal{H}(A, I)\left(B^{\prime}\right)$ is the subgroup in the group of $B^{\prime}$-linear Poisson derivations $D: A \otimes B^{\prime} \rightarrow(A \otimes I) \otimes B^{\prime}$ spanned by those derivations for which id $\oplus D: A_{B} \otimes B^{\prime} \rightarrow$ $A_{B} \otimes B^{\prime}$ is compatible with restricted structures. This group of derivations coincides with $\Omega_{c l}^{1}(A)\left(B^{\prime}\right)$ (unlike $\mathcal{H}(A, I)\left(B^{\prime}\right)$, it is not only a group, but also a $B^{\prime}$-module). We note that for any Poisson derivation $D: A \otimes B^{\prime} \rightarrow A \otimes I \otimes B^{\prime}$, the composition $K \circ D$ is a Frobenius derivation. By Theorem 1.12, a map is compatible with a 
restricted structure if and only if it preserves the associated isomorphism

$$
\left.C_{*}^{1}\left[\mathcal{M}_{\Omega}\right] \cong \Omega\right\lrcorner\left[\theta_{K}\right] .
$$

We leave it to the reader to check that in terms of $D$, this can be rewritten as

$$
\left.\left.C^{1}(\Omega\lrcorner D\right)=\Omega\right\lrcorner(K \circ D),
$$

which coincides with (3.2).

Lemma 3.11. Assume given a quantization base $B$ with maximal ideal $\mathfrak{m}_{B}$ such that $\mathfrak{m}_{B}^{2}=0$. Then the natural surjective group homomorphism $\mathrm{G}^{B} \rightarrow \mathrm{H}$ obtained by reduction $\bmod \mathfrak{m}_{B}$ admits a splitting $\mathrm{H} \rightarrow \mathrm{G}^{B}$.

Proof. If $h=0$, so that $A_{B}$ is a Poisson algebra, the claim is obvious: $\mathrm{H}$ naturally acts on $A_{B}=A \otimes_{k} B$. Denote by $B^{\prime}$ the associated graded quotient of the algebra $B$ with respect to the $h$-adic filtration on $B$. We claim that $\mathrm{G}^{B} \cong \mathrm{G}^{B^{\prime}}$.

Indeed, since $k\langle 1\rangle$ is injective in the category of small Dieudonné modules, there exists a splitting $I=\mathfrak{m}_{B} \cong k \cdot h \oplus(I / h I)$. Therefore there exists a restricted algebra isomorphism $B \cong B^{\prime}$. Let $\iota: B \rightarrow B$ be the involution that fixes $B / h B$ and sends $h$ to $-h$, and let $\iota^{*} A_{B}^{o p p}$ be the opposite algebra to the $B$-quantization $A_{B}$ with the $B$-module structure twisted by $\iota$, and the same Poisson bracket and $p$-th power operation. Then it is easy to check that $\iota^{*} A_{B}^{o p p}$ is also a $B$-quantization of the algebra $A$. Moreover, since $\iota$ is identical on $B_{0}=B / k \cdot h$, both $A_{B}$ and $\iota^{*} A_{B}^{o p p}$ are objects in $Q\left(A_{0}, B\right)$, where $A_{0}=A_{B} / A \cdot h$. By Proposition 3.8, there exists an isomorphism $\iota: \iota^{*} A_{B}^{\text {opp }} \cong A_{B}$ identical on the quotient $A_{0}$. In other words, we have a natural lifting $\iota: A_{B} \rightarrow A_{B}$ of the map $\iota: B \rightarrow B$ to an anti-multiplicative map of restricted quantized algebras. The map $\iota^{2}: A_{B} \rightarrow A_{B}$ is then an automorphism of the algebra $A_{B}$ which is also identical on $A_{0}$. Since $\iota^{2}$ commutes with $\iota$, while $\iota$ is identical on $A_{0}$ and equal to ( - id) on $A \cdot h \subset A_{B}$, we must have $\iota^{2}=$ id, so that $\iota: A_{B} \rightarrow A_{B}$ is an anti-involution. Taking its eigenspace decomposition, we obtain a vector space isomorphism $A_{B} \cong A_{B}^{\prime}$. It is easy to check that this isomorphism is compatible with the Poisson bracket and the $p$-th power operation, while the products in $A_{B}$ and $A_{B}^{\prime}$ are related by

$$
a \cdot b=a b+h\{a, b\}
$$

(here $a \cdot b$ is the product in $A_{B}$, and $a b$ is the product in $A_{B}^{\prime}$ ). We conclude that every $B$-linear automorphism of the restricted quantized algebra $A_{B}$ fixes the product in $A_{B}^{\prime} \cong A_{B}$, and vice versa. Therefore we indeed have $\mathrm{G}_{B} \cong \mathrm{G}_{B}^{\prime}$.

Remark 3.12. In the case when $B=k[[h]]$ and $A_{B}=D$, the reduced Weyl algebra, it is easy to compute the successive quotients in the natural $h$-adic filtration on $\mathrm{G}=\mathrm{G}^{B}$ directly, without recourse to restricted structures and deformation theory. Namely, one first proves that $D\left(h^{-1}\right)$ is an Azumaya algebra over $k((h))$. Then by the Scolem-Noether Theorem all $h$-linear automorphisms of the algebra $D\left(h^{-1}\right)$ are inner. It is an easy exercise to check that an automorphism preserves $D \subset D\left(h^{-1}\right)$ and acts trivially on $A=D / h D$ if and only if it is given by conjugation with an invertible element in $D \subset D\left(h^{-1}\right)$. One concludes that the kernel $\mathrm{G}^{>0}$ of the natural map $\mathrm{G} \rightarrow \mathrm{H}$ is isomorphic to $D^{*} / k[[h]]^{*}$, where $D^{*}$ is the group of invertible elements in $D$; the isomorphism in Lemma 3.10 immediately follows by applying the 
$d \log$ map. The problem with this approach is that it is not clear how to generalize it to non-trivial quantization bases $B$, and it is probably not possible at all to use it to prove Lemma 3.11 .

\section{Formal GEOMETRY}

In order to obtain global results about quantizations, we use the technique of the so-called formal geometry. This is similar to the approach in [BK1, 3.1] in the characteristic 0 case; however, the situation in positive characteristic is somewhat simpler, and in particular, all the algebraic groups we use are of finite type.

4.1. The setup. Fix a finite-dimensional vector space $W$ over $k$, and let $A=$ $k\left[W^{*}\right] / \mathfrak{m}^{[p]}$ be the commutative $k$-algebra associated to $W$ in Subsection 3.1. Recall that we have the algebraic group Aut $A$ of all automorphisms of the commutative algebra $A$ and the reduced subgroup (Aut $A)_{0} \subset$ Aut $A$ of automorphisms which preserve the maximal ideal $\mathfrak{m} \subset A$. The quotient (Aut $A) /(\text { Aut } A)_{0}$ is naturally identified with the spectrum $\operatorname{Spec} A$ of the algebra $A$-indeed, the action of Aut $A$ on $\operatorname{Spec} A$ is transitive, and (Aut $A)_{0} \subset$ Aut $A$ is the stabilizer of the closed point.

Formal geometry is based on the following observation. Let $X$ be a manifold over $k,-$ that is, a quasiregular reduced scheme $X / k$ of finite type, - and assume that $\operatorname{rk}(\mathcal{T}(X))=\operatorname{dim} W$. Then there exists a canonical (Aut $A)_{0}$-torsor $\mathcal{M}_{X}$ over $X$. Namely, for any affine $S / k$ we define

$$
\mathcal{M}_{X}(S)=\operatorname{Hom}_{\text {ét }}(\operatorname{Spec} A \times S, X \times S) \text {, }
$$

the set of all unramified maps $\varphi$ : Spec $A \times S \rightarrow X \times S$ of schemes over $S$. To check that the functor $\mathcal{M}_{X}(S)$ is represented by a faithfully flat scheme over $X$ is an easy exercise left to the reader. The group Aut $A$ acts on $\mathcal{M}_{X}$ through its action on $\operatorname{Spec} A$; a second easy check shows that the action is effective, and that $X \cong \mathcal{M}_{X} /(\text { Aut } A)_{0}$.

Informally, $\mathcal{M}_{X}$ is the scheme of all pairs $\langle x, \varphi\rangle$ of a point $x \in X$ and a surjective map $\mathcal{O}_{X, x} \rightarrow A-$ a "coordinate system in the Frobenius neighborhood of $x \in X$ ". We will call $\mathcal{M}_{X}$ the torsor of Frobenius frames on $X$. The projection $\mathcal{M}_{X} \rightarrow X=$ $\mathcal{M}_{X} /(\text { Aut } A)_{0}$ corresponds to forgetting the coordinate system. We note that the torsor of Frobenius frames is locally trivial in Zariski topology on $X$.

The action of the whole group Aut $A$ on $X$ is also effective, and the quotient $\mathcal{M}_{X} /($ Aut $A)$ is naturally identified with $X^{[p]}$, that is, $X$ with structure sheaf $\mathcal{O}_{X}^{p} \subset$ $\mathcal{O}_{X}$. All in all, we have a diagram

$$
\mathcal{M}_{X} \longrightarrow X \stackrel{\mathrm{Fr}}{\longrightarrow} X^{[p]} .
$$

Here the left-hand side map is the quotient map $\mathcal{M}_{X} \rightarrow \mathcal{M}_{X} /(\text { Aut } A)_{0} \cong X$, the composition is the quotient map $\mathcal{M}_{X} \rightarrow \mathcal{M}_{X} /(\text { Aut } A)_{0} \cong X^{[p]}$, and the right-hand side map is the Frobenius map of the manifold $X$. The scheme $\mathcal{M}_{X}$ can be treated either as an (Aut $A)_{0}$-torsor over $X$, or as an Aut $A$-torsor over $X^{[p]}$.

Having said this, we can now describe various differential-geometric structures on $X$ in terms of reductions of the torsor $\mathcal{M}_{X}$ to various subgroups in Aut $A-$ or, more generally, its liftings to various algebraic groups $G$ equipped with a map $G \rightarrow$ Aut $A$. 
Definition 4.1. For any algebraic group $G$ over $k$ equipped with a map $\rho: G \rightarrow$ Aut $A$, a $G$-structure on the manifold $X$ is a $G$-torsor $\mathcal{M}_{G}$ over $X^{[p]}$ equipped with a map $\mathcal{M}_{G} \rightarrow \mathcal{M}_{X}$ compatible with $\rho$.

For any $k$-vector space $V$ equipped with an algebraic action of the group Aut $A$, we have the associated bundle $\operatorname{Loc}\left(\mathcal{M}_{X}, V\right)$; this defines an exact localization functor

$$
\text { Loc : }(\text { Aut } A)-\bmod ^{\mathrm{fg}} \rightarrow \operatorname{Coh}\left(X^{[p]}\right)
$$

from the category of finite-dimensional representations of the group Aut $A$ to the category of coherent sheaves of $\mathcal{O}_{X}^{p}$-modules on $X^{[p]}$. Essentially this is just the flat descent functor for the (Aut $A$ )-torsor $\mathcal{M}_{X}$. For example, the localization $\operatorname{Loc}(k)$ of the trivial (Aut $A$ )-module $k$ is isomorphic to the structure sheaf $\mathcal{O}_{X}^{p}$. More generally, assume given a commutative algebraic group $H$ over $k$ - equivalently, a sheaf of abelian groups on Spec $k$ in the flat topology - and assume that the group Aut $A$ acts on $H$ in an algebraic way. Then flat descent allows one to define a sheaf $\operatorname{Loc}\left(\mathcal{M}_{G}, H\right)$ of abelian groups on $X^{[p]}$ in flat topology. This works just as well for any $G$-structure $\mathcal{M}_{G}$ on $X$ - we have a localization functor $\operatorname{Loc}\left(\mathcal{M}_{G},-\right)$ from the category of abelian algebraic groups over $k$ equipped with a $G$-action to the category of sheaves of abelian groups on $X^{[p]}$ in the flat topology. We recall that both of these categories are abelian; the localization functor is exact.

Using localization, one defines obstruction theory for $G$-structures in the following way. Assume given an algebraic group $G$ over $k$ equipped with a map $G \rightarrow$ Aut $A$, and another algebraic group $G_{1}$ over $k$ equipped with a surjective map $G_{1} \rightarrow G$. Moreover, assume that the kernel $H$ of the map $G_{1} \rightarrow G$ is an abelian algebraic group. Then $G$ acts by conjugation on the group $H$, and this action is algebraic. The extension $G_{1} \rightarrow G$ is completely defined by a cohomology class $c \in H^{2}(G, H)$ (in particular, $G_{1}$ coincides with the semi-direct product $H \rtimes G$ if and only if $c=0)$. Assume given a $G$-structure $\mathcal{M}$ on a manifold $X$ of $\operatorname{dimension} \operatorname{dim} X=\operatorname{dim} W$. Then we can apply localization to the class $c \in H^{2}(G, H)=\operatorname{Ext}^{2}\left(\mathbb{G}_{a}, H\right)$ and obtain a cohomology class

$$
\operatorname{Loc}(\mathcal{M}, c) \in \operatorname{Ext}^{2}\left(\mathcal{O}_{X}^{p}, \operatorname{Loc}(\mathcal{M}, V)\right)=H^{2}\left(X^{[p]}, \operatorname{Loc}(\mathcal{M}, H)\right) .
$$

The following is the standard obstruction theory statement; for the proof see, e.g., $[\mathrm{G}]$.

Lemma 4.2. The $G$-torsor $\mathcal{M}$ admits a restriction to a $G_{1}$-torsor $\mathcal{M}_{1}$ if and only if $\operatorname{Loc}(\mathcal{M}, c)=0$. Moreover, if this happens, then for any two such restrictions $\mathcal{M}_{1}, \mathcal{M}_{1}^{\prime}$ we have a well-defined class $\left[\mathcal{M}_{1}-\mathcal{M}_{1}^{\prime}\right] \in H^{1}\left(X^{[p]}, \operatorname{Loc}(\mathcal{M}, H)\right)$, and $\mathcal{M}_{1} \cong \mathcal{M}_{1}^{\prime}$ if and only if $\left[\mathcal{M}_{1}-\mathcal{M}_{1}^{\prime}\right]=0$.

4.2. The proofs. We can now prove Proposition 1.18, Proposition 1.19 and Proposition 1.24 .

Lemma 4.3. Let $B$ be a quantization base over the field $k$, let $X$ be a symplectic manifold of dimension $\operatorname{dim} X=\operatorname{dim} W$, and let $\mathrm{G}^{B}$ be the automorphism group of the regular $B$-quantization $A_{B}$ of the algebra $A$ introduced in Proposition 3.8. Then there is a natural equivalence between the category of regular $B$-quantizations of $X$ and the category of $\mathrm{G}^{B}$-structures on $X$. Moreover, every $\mathrm{G}^{B}$ structure $\mathcal{M}_{G}$ on $X$ is locally trivial in étale topology. 
Proof. Given a $\mathrm{G}^{B}$-structure $\mathcal{M}_{B}$ on $X$, we obtain a regular $B$-quantization $\mathcal{O}_{B}$ as

$$
\mathcal{O}_{B}=\operatorname{Loc}\left(\mathcal{M}_{B}, A_{B}\right) \text {. }
$$

Conversely, given a regular $B$-quantization $\mathcal{O}_{B}$, we take $\mathcal{M}_{B}$ to be the set of pairs,

$$
\mathcal{M}=\left\{\langle x, \varphi\rangle \mid x \in X^{[p]}, \varphi:\left(\mathcal{O}_{B}\right)_{x} \rightarrow A_{B}\right\}
$$

where $x \in X^{[p]}$ is a point, and $\varphi$ is an isomorphism between the stalk $\left(\mathcal{O}_{B}\right)_{x}$ of the $\mathcal{O}_{X}^{p}$-module sheaf $\mathcal{O}_{B}$ at the point $x$ and the standard restricted quantized algebra $A_{B}$. One easily checks that $\mathcal{M}_{B}$ has a natural algebraic structure; the group $\mathrm{G}^{B}$ acts on $\mathcal{M}_{B}$ by $g(\langle x, \varphi\rangle)=\langle x, g \circ \varphi\rangle$, this action is effective, and the natural projection $\mathcal{M}_{B} \rightarrow X^{[p]}$ identifies $\mathcal{M}_{B} / G^{B}$ with an open subset in $X^{[p]}$. Finally, by Proposition 3.8 the algebra $\left(\mathcal{O}_{B}\right)_{x}$ is isomorphic to $A_{B}$ for any $x \in X^{[p]}$, so that $\mathcal{M}_{B} / \mathrm{G}^{B}$ is the whole $X^{[p]}$, and $\mathcal{M}_{B}$ is indeed a $\mathrm{G}^{B}$-structure on $X$. To prove the second claim, we note that by Lemma 3.10 the kernel of the map $\mathrm{G}^{B} \rightarrow$ Aut $A$ is an iterated extension of smooth commutative algebraic groups of the form $\mathcal{H}\langle A, I\rangle$. Therefore the torsor $\mathcal{M}_{B}$ over $\mathcal{M}_{X}$ is locally trivial in étale topology (see, e.g., [M, III, §3, Theorem 3.9]). But $\mathcal{M}_{X}$ is in turn locally trivial in étale - and even in Zariski - topology on $X$.

Proof of Proposition 1.18 and Proposition 1.19. Proposition 1.18 is a direct combination of Lemma 4.3, Lemma 4.2 and Lemma 3.10. To deduce Proposition 1.19, it suffices to add Lemma 3.11 to the mix.

Proof of Proposition 1.24. The first claim follows from Lemma 3.2. To prove the second claim, we note that by Lemma 4.3, the algebra sheaf $\mathcal{O}_{h}$ is the associated bundle to a $\mathrm{G}$-torsor $\mathcal{M}_{h}$ on $X^{(1)}$, where $\mathrm{G}=\operatorname{Aut}(D)$ is the automorphism group of the reduced Weyl algebra $D$. The group $\mathrm{G}$ is in fact obtained by Weil restriction of scalars from a group $\widehat{\mathrm{G}}$ over the Taylor power series algebra $k[[h]]$, and the torsor $\mathcal{M}_{h}$ is obtained by restriction of scalars from a torsor $\widehat{\mathcal{M}}_{h}$ over the scheme $\widehat{X}$. Over $\bar{X}=\widehat{X} \backslash X^{(1)} \subset \widehat{X}$, the torsor $\widehat{\mathcal{M}}_{h}$ is a torsor over the group $\overline{\mathrm{G}}=\widehat{\mathrm{G}} \otimes_{k[[h]]} k((h))$. However, we have

$$
\overline{\mathrm{G}} \cong \operatorname{Aut}\left(D \otimes_{k[[h]]} k((h))\right) \cong P G L\left(p^{\frac{\operatorname{dim} X}{2}}, k((h))\right),
$$

and the torsor $\widehat{\mathcal{M}}_{h}$ over $\bar{X}$ becomes exactly the principal bundle associated to the Azumaya algebra $\mathcal{O}_{h}\left(h^{-1}\right)$. Its class $\overline{\operatorname{Br}\left(\left[\mathcal{O}_{h}\right]\right)} \in \operatorname{Br}(\bar{X})$ is represented by a gerb bound by $\mathcal{O}_{\bar{X}}^{*}$ which is associated to the torsor $\widehat{\mathcal{M}}_{h}$ and the standard central extension

$$
k((h))^{*} \longrightarrow G L\left(p^{\frac{\operatorname{dim} X}{2}}, k((h))\right) \longrightarrow P G L\left(p^{\frac{\operatorname{dim} X}{2}}, k((h))\right) .
$$

Since after restriction to $\widehat{G} \subset \bar{G}$ this extension reduces to a central extension of the group $\widehat{\mathrm{G}}$ by $k[[h]]^{*}$, the gerb representing $\overline{\operatorname{Br}\left(\left[\mathcal{O}_{h}\right]\right)}$ extends to a gerb on $\widehat{X}$ bound by $\mathcal{O}_{\widehat{X}}^{*}$, so that the class $\overline{\operatorname{Br}\left(\left[\mathcal{O}_{h}\right]\right)}$ comes from a class $\left.\widehat{\operatorname{Br}\left(\left[\mathcal{O}_{h}\right]\right.}\right) \in \operatorname{Br}(\widehat{X})$. However, by assumption $H^{2}\left(X^{(1)}, \mathcal{O}_{X}^{p}\right)=0$; considering the spectral sequence associated to the $h$-adic filtration on $\mathcal{O}_{\widehat{X}}^{*}$, we conclude that the restriction map

$$
\operatorname{Br}(\widehat{X}) \rightarrow \operatorname{Br}\left(X^{(1)}\right)
$$


is an isomorphism. To finish the proof, it suffices to check that the restriction of the class $\left.\widehat{\operatorname{Br}\left(\left[\mathcal{O}_{h}\right]\right.}\right)$ to $X^{(1)} \subset \widehat{X}$ indeed coincides with $\operatorname{Br}\left(\left[\mathcal{O}_{h}\right]\right)$. This immediately follows from the commutative diagram

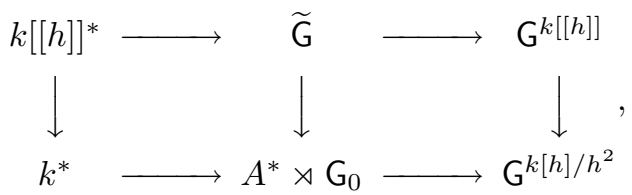

where the rows are central extensions of algebraic groups, and $\widetilde{G}$ is the extension induced by (4.1).

Remark 4.4. As the above proof shows, there is no canonical way to extend the Azumaya algebra $\mathcal{O}_{h}\left(h^{-1}\right)$ itself to an Azumaya algebra on $\widehat{X}$ - it is only its class in the Brauer group that has such an extension. The reason for that is clear from the group theoretic description: Aut $(D)$ and $P G L\left(p^{\frac{\operatorname{dim} X}{2}}, k[[h]]\right)$ are two different parabolic subgroups in the loop group $P G L\left(p^{\frac{\operatorname{dim} X}{2}}, k((h))\right)$, and both are maximal parabolic. Extending the Azumaya algebra would correspond to finding an embedding $\operatorname{Aut}(D) \subset P G L\left(p^{\frac{\operatorname{dim} X}{2}}, k[[h]]\right)$; since both are maximal parabolic, such an embedding does not exist.

\section{ACKNOWLEDGMENTS}

This paper is a part of the ongoing project for the systematic use of quantizations in positive characteristic to obtain information about algebraic symplectic varieties. The project has already been going on for several years, and during this time we have benefited from the discussions and help from many people. Our original encouragement came from the late A.N. Tyurin, who is very much missed. We would like to thank A. Beilinson, A. Bondal, V. Drinfeld, T. Ekedal, M. Emerton, P. Etingof, B. Feigin, M. Finkelberg, V. Ginzburg, D. Huybrechts, D. Kazhdan, M. Kontsevich, A. Kuznetsov, M. Lehn, Ch. Sorger, D. Van Straten, M. Verbitsky and V. Vologodsky for much help and much valuable input. During the preparation of this paper, we had the opportunity to work at several first-rate mathematical institutions whose hospitality is gratefully acknowledged; in particular, we would like to thank Northwestern University, the University of Chicago, Université Paris 7, Université de Nantes, Universität Gutenberg at Mainz, Hebrew University of Jerusalem and the Mittag-Laefller Institute in Stockholm.

\section{REFERENCES}

[BK1] R. Bezrukavnikov and D. Kaledin, Fedosov quantization in algebraic context, Moscow Math. J. 4 (2004), 559-592. MR2119140 (2006j:53130)

[BK2] R. Bezrukavnikov and D. Kaledin, McKay equivalence for symplectic quotient singularities, Proc. of the Steklov Inst. of Math. 246 (2004), 13-33. MR2101282 (2006e:14006)

[BMR] R. Bezrukavnikov, I. Mirković, and D. Rumynin, Localization of modules for a semisimple Lie algebra in prime characteristic, math.RT/0205144.

[D] M. Demazure, Lectures on p-Divisible Groups, Lecture Notes in Math. 302, Springer, Berlin-Heidelberg-New York, 1972. MR0344261 (49:9000)

[DP] M. Demazure and P. Gabriel, Groupes Algébriques. Tome I: Géométrie algébrique, généralités, groupes commutatifs, North-Holland Publishing Co., Amsterdam, 1970. MR0302656 (46:1800)

[G] J. Giraud, Cohomologie non abélienne, Die Grundlehren der mathematischen Wissenschaften, Band 179, Springer-Verlag, Berlin-New York, 1971. MR0344253 (49:8992) 
[EGA] A. Grothendieck, Éléments de Géométrie Algébrique, III, Publ. Math. IHES 24.

[K] M. Kontsevich, Deformation quantization of algebraic varieties, Lett. Math. Phys. 56 (2001), 271-294. MR1855264 (2002j:53117)

[M] J. Milne, Étale cohomology, Princeton Math. Series, 33, Princeton U. Press, 1980. MR559531 (81j:14002)

[NT] R. Nest and B. Tsygan, Deformations of symplectic Lie algebroids, deformations of holomorphic symplectic structures, and index theorems, Asian J. Math. 5 (2001), 599-635. MR1913813 (2003k:53126)

[Y] A. Yekutieli, On Deformation Quantization in Algebraic Geometry, Adv. in Math. 198 (2006), 383-432. MR2183259 (2006j:53131)

Department of Mathematics, Massachusets Institute of Technology, Cambridge, MassachusetTs 02139

E-mail address: bezrukav@math.mit.edu

Steklov Institute, Gubkina 8, Moscow, 119991, Russia

E-mail address: kaledin@mccme.ru 